\begin{tabular}{|c|c|}
\hline $\begin{array}{l}\text { 2. To: (Receiving organization) } \\
\text { Distribution }\end{array}$ & $\begin{array}{l}\text { 3. From: (Originating organization) } \\
\text { NHC Process Chemistry }\end{array}$ \\
\hline $\begin{array}{l}\text { 5. Proj./Prog./Dept./Div.: } \\
\text { TWRS }\end{array}$ & $\begin{array}{l}\text { 6. Design Authority/ Design Agent/Cog } \\
\text { Engr.: } \\
\text { D. L. Herting }\end{array}$ \\
\hline
\end{tabular}

8. Originator Remarks:

FY 1999 Report for TTP No. RL0-8-WT-41, Tanks Focus Area (EM-50)

11. Receiver Remarks: 11A. Design Baseline Document? [] Yes [X] No

\section{Related EDT No.: \\ NA \\ 7. Purchase order No.: \\ NA}

9. Equip./Component No.:

NA

10. System/Bldg./Facility: 222-S Laboratory

12. Major Assm. Dwg. No.:

NA

13. Permit/Permit Application No.: NA

14. Required Response Date:

September 30, 1998

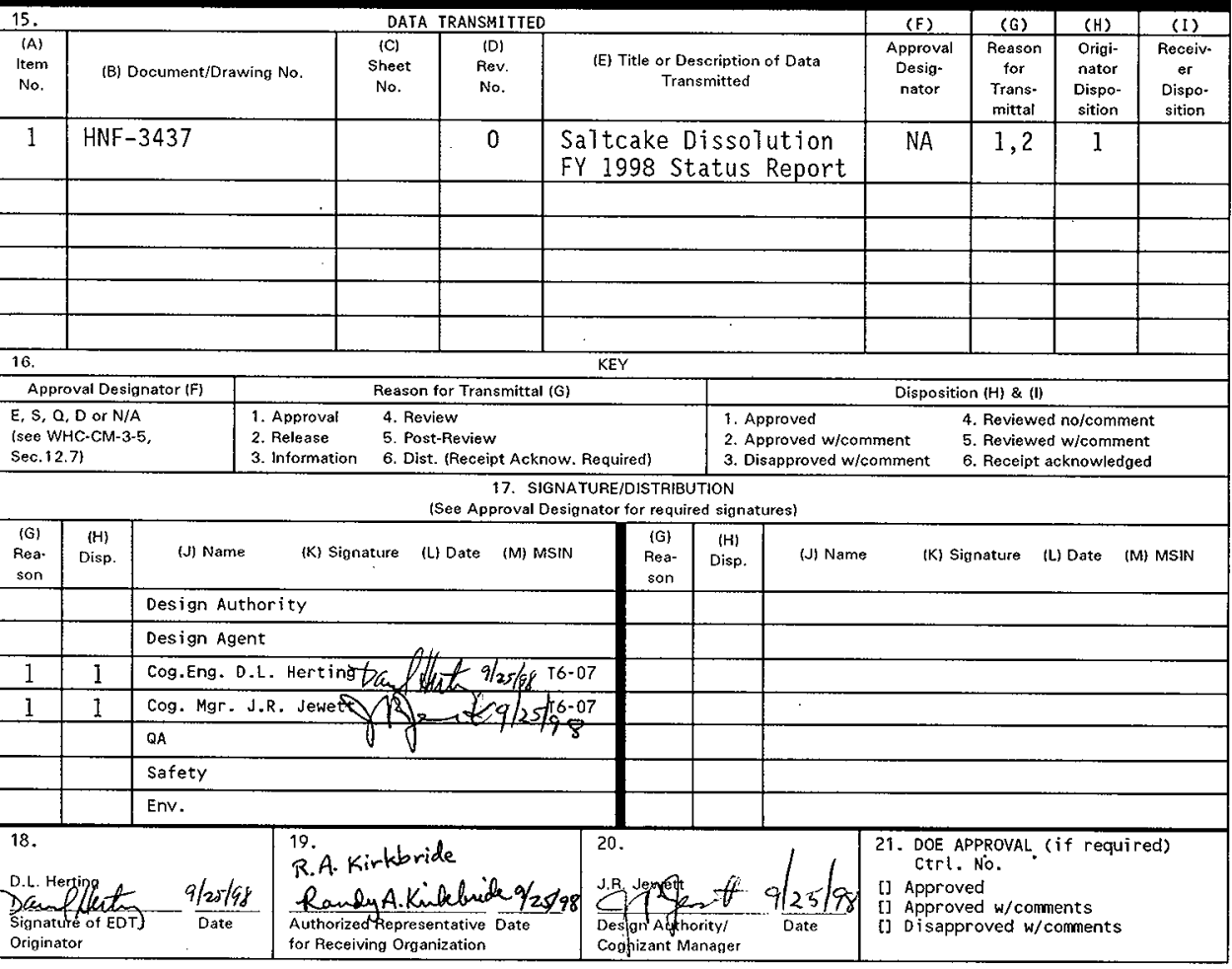




\title{
Saltcake Dissolution FY 1998 Status Report
}

\author{
D. L. Herting \\ D. W. Edmonson \\ Numatec Hanford Corporation
}

Date Published

September 1998

Prepared for the U.S. Department of Energy

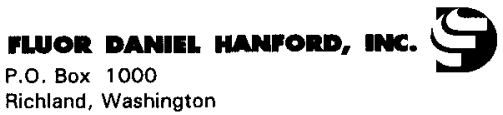

Richland, Washington

Hanford Management and integration Contractor for the

U.S. Department of Energy under Contract DE-AC06-96RL13200 
LEGAL DISCLAIMER

This report was prepared as an account of work sponsored by an agency of the United States Government. Neither the United States Government nor any agency thereof, nor any of their employees, nor any of their contractors, subcontractors or their employees, makes any warranty, express or implied, or assumes any legal liability or responsibility for the accuracy, completeness, or any third party's use or the results of such use of any information, apparatus, product, or process disclosed, or represents that its use would not infringe privately owned rights. Reference herein to any specific commercial product, process, or service by trade name, trademark, manufacturer, or otherwise, does not necessarily constitute or imply its endorsement, recommendation, or favoring by the United States Government or any agency thereof or its contractors or subcontractors. The views and opinions of authors expressed herein do not

necessarily state or reflect those of the United States Government or any agency thereof.

This report has been reproduced from the best available copy.

Available in paper copy and microfiche.

Available to the U.S. Department of Energy

and its contractors from

U.S. Department of Energy

Office of Scientific and Technical Information (OSTI)

P.O. Box 62

Oak Ridge, TN 37831

(615) $576-8401$

Available to the public from the U.S. Department of Commerce

National Technical Information Service (NTIS)

5285 Port Royal Road

Springfield, VA 22161

(703) $487-4650$

Printed in the United States of America

DISCLM-1.CHP (8-95) 


\title{
Saltcake Dissolution FY 1998 Status Report
}

\author{
D. L. Herting \\ D. W. Edmonson \\ Numatec Hanford Corporation, Richland, WA 99352 \\ U.S. Department of Energy Contract DE-AC06-96RL13200
}

$\begin{array}{lll}\text { EDT/ECN: } & 625405 & \text { UC: } 2030 \\ \text { Org Code: } & 8 C 510 & \text { Charge Code: W8521 } \\ \text { B\&R Code: } & \text { EW3130010 } & \text { Tota7 Pages: } 61\end{array}$

Key Words: BY-102, BY-106, B-106, ESP, Retrieval, Sodium, Nitrate, Fluoride, Phosphate, Sulfate, Carbonate

Abstract: A laboratory scouting study was completed on the dissolution characteristics of Hanford waste from three single-shell waste tanks: 241-BY-102, 241-BY-106, and 241-B-106. Gross dissolution behavior (percent undissolved solids as a function of dilution) is explained in terms of characteristics of individual salts in the waste. The percentage of the sodjum inventory retrievable from the tanks by dissolving saltcake at reasonable dilution levels is estimated at $86 \%$ of the total sodium for tank BY-102, 98\% for BY-106, and $79 \%$ for B-106.

TRADEMARK DISCLAIMER. Reference herein to any specific commercial product, process, or service by trade name, trademark, manufacturer, or otherwise, does not necessarily constitute or imply its endorsement, recommendation, or favoring by the United States Government or any agency thereof or its contractors or subcontractors.

Printed in the United States of America. To obtain copies of this document contact: Document Control Services, P.0. Box 950, Mailstop H6-08, Richland WA 99352, Phone (509) 372-2420;

Fax (509) 376-4989.
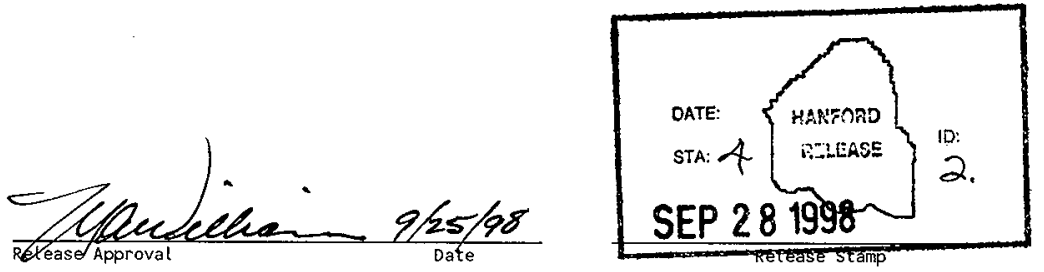


\section{CONTENTS}

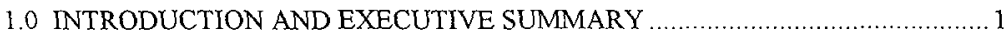

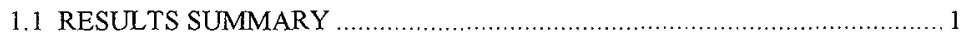

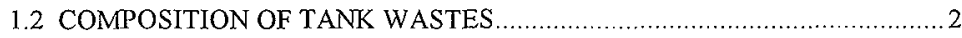

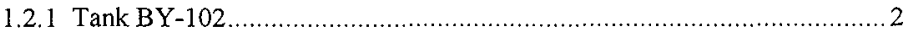

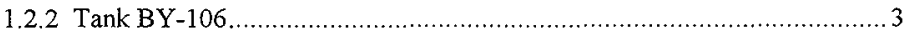

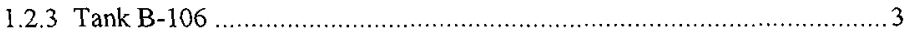

2.0 DESCRIPTION OF LABORATORY STUDIES ............................................. 5

2.1 PREPARATION OF COMPOSITE SAMPLES ….................................... 5

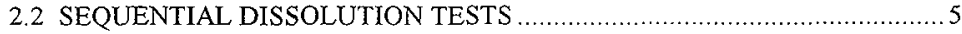

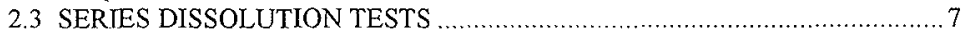

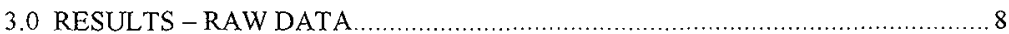

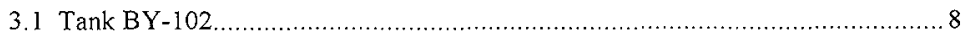

3.1.1 BY-102 Sequential Dissolution Test ............................................. 8

3.1.2 BY-102 Series Dissolution Test, Ambient Temperature …................... 8

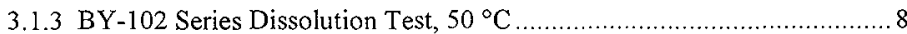

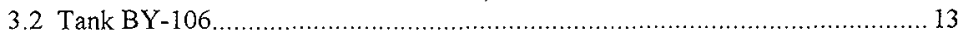

3.2.1 BY-106 Sequential Dissolution Test ............................................ 13

3.2.2 BY-106 Series Dissolution Test, Ambient Temperature........................ 13

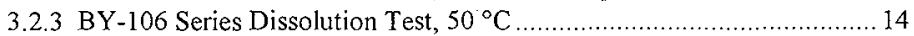

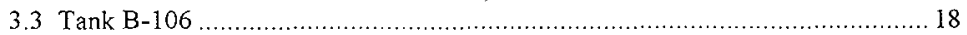

3.3.1 B-106 Sequential Dissolution Test .................................................. 18

4.0 DILUTION RESULTS, VOLUME AND WEIGHT MEASUREMENTS ..............22

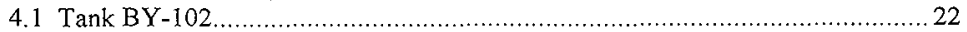

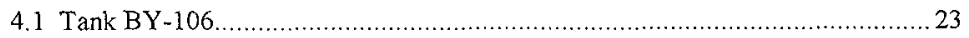

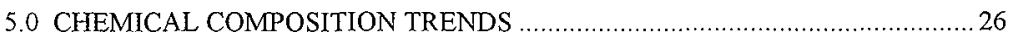

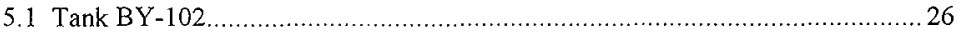

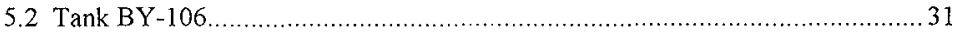

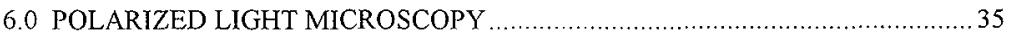

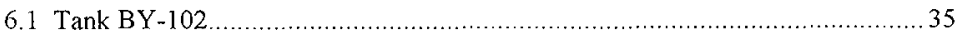

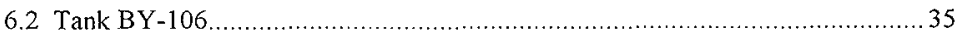

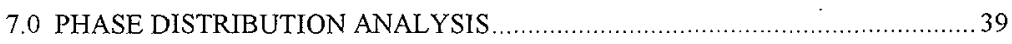

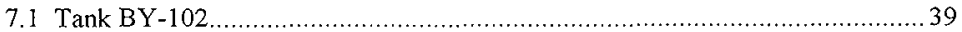

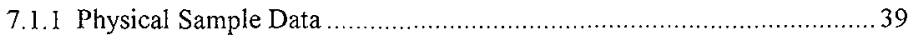

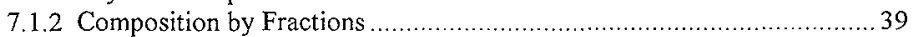

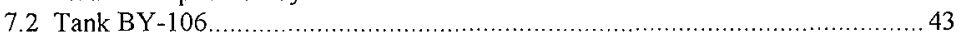

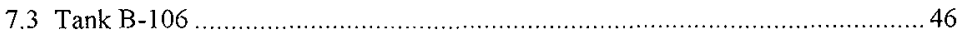




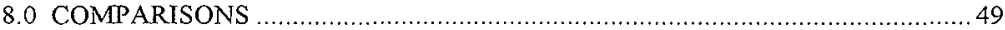

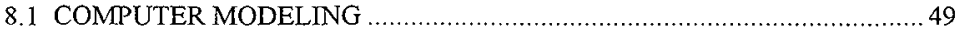

8.2 COMPARISONS WITH TCR DATA AND SLUDGE WASH DATA …........50

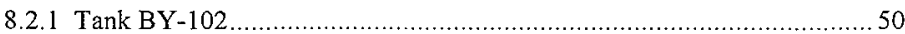

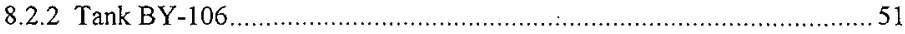

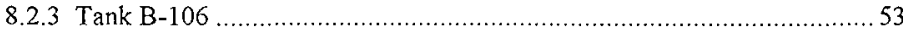

9.0 References 
HNF-3437, Rev. 0

\section{TABLES}

2-1 Segment Samples Used to Create Core Composite Samples ................. 6

3-1 Raw Data, Tank BY-102 Sequential Dissolution Test ...................... 9

3-2 Analytical Results, Tank BY-102 Sequential Dissolution Test f............. 10

3-3 Raw Data, Tank BY-102 Series Dissolution Test, Ambient Temperature .... 11

3-4 Analytical Results, Tank BY-102 Series Dissolution Test, Ambient Temp ... 11

3-5 Raw Data, Tank BY-102 Series Dissolution Test, $50{ }^{\circ} \mathrm{C} \ldots \ldots \ldots \ldots \ldots \ldots \ldots . \ldots 12$

3-6a Analytical Results, Tank BY-102 Series Dissolution Test, $50^{\circ} \mathrm{C}$ (diluted) ... $\quad 12$

3-6b Analytical Results, Tank BY-102 Series Dissolution Test, $50^{\circ} \mathrm{C}$ (undiluted) 13

3-7 Raw Data, Tank BY-106 Sequential Dissolution Test ...................... 14

3-8 Analytical Results, Tank BY-106 Sequential Dissolution Test ............. 15

3-9 Raw Data, Tank BY-106 Series Dissolution Test, Ambient Temperature ... 16

3-10 Analytical Results, Tank BY-106 Series Dissolution Test, Ambient Temp ... 16

3-11 Raw Data, Tank BY-106 Series Dissolution Test, $50{ }^{\circ} \mathrm{C} \ldots \ldots \ldots \ldots \ldots \ldots \ldots 17$

3-12 Analytical Results, Tank BY-106 Series Dissolution Test, $50^{\circ} \mathrm{C} \ldots \ldots \ldots \ldots 17$

3-13 Raw Data, Tank B-106 Sequential Dissolution Test ....................... 19

3-14 Analytical Results, Tank B-106 Sequential Dissolution Test ............... 20

3-15 Raw Data, Tank B-106 Series Dissolution Test, Ambient Temperature ..... 21

4-1 Percent Centrifuged Solids as a Function of Dilution, Tank BY-102 _...... 22

4-2 Percent Centrifuged Solids as a Function of Dilution, Tank BY-106 ....... 24

5-1 Fluoride Concentrations Predicted from Phosphate and Sulfate Data ....... 30

7-1 Summary of Measurements from BY-102 Sequential Dissolution Test ..... 39

7-2 Tank BY-102 Analysis by Fractions .................................. 40

7-3 Tank BY-102 Solid/Liquid Phase Distribution at 100\% Dilution ........... 42

7-4 Summary of Measurements from BY-106 Sequential Dissolution Test ..... 43

7-5 Tank BY-106 Analysis by Fractions ................................... . 44

7-6 Tank BY-106 Solid/Liquid Phase Distribution at 100\% Dilution .......... 45

7-7 Summary of Measurements from B-106 Sequential Dissolution Test ....... 46

7-8 Tank B-106 Analysis by Fractions ...................................... 47

7-9 Tank B-106 Solid/Liquid Phase Distribution at 100\% Dilution ............ 48

8-1 Tank BY-102 Comparison with TCR Data ............................ 51

8-2 Tank BY-106 Comparison with TCR Data ................................ 52

8-3 Tank B-106 Comparison with TCR Data ............................. 54

8-4 Percentage of B-106 Analyte Undissolved in Inhibited Water .............. 55 


\section{FIGURES}

4-1 Tank BY-102 Saltcake Dissolution .................................. 23

4-2 Tank BY-106 Saltcake Dissolution ................................. 25

5-1 Tank BY-102 Chloride, Fluoride, Oxalate ........................... 27

5-2 Tank BY-102 Nitrite, TIC ........................................ 28

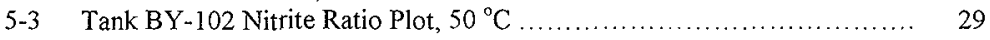

5-4 Tank BY-102 Phosphate, Sulfate ................................. 29

5-5 Tank BY-106 Nitrate Concentrations .............................. 31

5-6 Tank BY-106 Nitrite, TIC ..................................... 32

5-7 Tank BY-106 Fluoride, Phosphate, Sulfate ......................... 33

5-8 Tank BY-106 Chloride, Oxalate ................................ 34

6-1 Crystals Formed when BY-102 Liquid Cooled ... Smaller Crystals ........ 36

6-2 Crystals Formed when BY-102 Liquid Cooled ... Larger Crystals ......... 37

6-3 Crystals Formed by Evaporation of BY-106 Liquid from $2^{\text {nd }}$ Water Wash .. 38

\section{ACRONYMS AND ABBREVIATIONS}

B-106 Single-shell tank 24l-B-106

BSltCk Saltcake deposited during 242-B Evaporator operation, 1951 to 1953

BY-102 Single-shell tank 241-BY-102

BY-106 Single-shell tank 241-BY-106

BYSltCk Saltcake deposited during ITS evaporator campaigns

ESP

GEA

IC

ICP

ITS

kgal

$\mathrm{kL}$

ML

PLM

rpm

TCR

TIC

Environmental Simulation Program

gamma energy analysis

ion chromatography

inductively coupled plasma spectroscopy

in-tank solidification

kilogallon (1,000 gallons)

kiloliter (1,000 liters)

megaliter ( $1,000,000$ liters $)$

polarized light microscope (or microscopy)

revolutions per minute

Tank Characterization Report

total inorganic carbon

TOC total organic carbon

TWRS Tank Waste Remediation System 


\subsection{INTRODUCTION AND EXECUTIVE SUMMARY}

A laboratory study was completed on the dissolution characteristics of Hanford waste from three single-shell waste tanks: 241-BY-102, 241-BY-106, and 241-B-106 (henceforth referred to as BY-102, BY-106, and B-106). The first two tanks contain saltcake waste; the third tank was predicted to contain saltcake, based on historical process information (Agnew 1997), but the waste behaved more like a sludge. For this report, saltcake is defined as tank waste composed mainly of water-soluble sodium salts and is relatively dry; sludge is defined as tank waste that contains a high proportion of water-insoluble metal hydroxides and other water-insoluble compounds, and has a relatively high moisture content.

The Tank Waste Remediation System (TWRS) is tasked with retrieving waste from double-shell and single-shell tanks to provide feed for vitrification. The TWRS organization needs chemical and physical data to evaluate technologies for retrieving the waste. No significant laboratory studies have been done to evaluate in-tank dissolution parameters for the various types of saltcake wastes that exist in single-shell tanks.

A computer modeling program known as the Environmental Simulation Program (ESP) is being used by the TWRS organization to predict dilution/retrieval behavior of all tank waste types. Data from this task is provided to ESP users to support evaluation, refinement, and validation of the ESP model.

This work was funded by the Tanks Focus Area (EM-50) under Technical Task Plan Number RL0-8-WT-4I, "PHMC Pretreatment - Saltcake Dissolution". The work will continue into fiscal year 1999 .

\subsection{RESULTS SUMMARY}

Weight percent dilution is defined in this report as 100 times the weight of diluent divided by the weight of undiluted sample. A water dilution of $50 \%$, for example, would be $50 \mathrm{~g}$ of $\mathrm{H}_{2} \mathrm{O}$ added to $100 \mathrm{~g}$ of undiluted sample.

Some of the dissolution data are plotted in Figures 4-1 and 4-2 as "Percent Undissolved" vs. weight percent dilution at two temperatures. The Percent Undissolved is defined as 100 times the weight of centrifuged solids after dissolution divided by the initial sample weight before diluent was added. This measurement provides a gross view of the extent of saltcake dissolution at various conditions, and is a rough measure of the fraction of waste that could be retrieved from the tank by pumping the supernatant liquid.

The percent undissolved solids for tanks BY-102, BY-106, and B-106 at $25^{\circ} \mathrm{C}$ and $100 \%$ dilution with water were $75 \%, 27 \%$, and $50 \%$, respectively. At $100 \%$ dilution and $50{ }^{\circ} \mathrm{C}$, the dissolution improved to $48 \%$ and $19 \%$ for $\mathrm{BY}-102$ and $\mathrm{BY}-106$ (B-106 was not measured). At $300 \%$ dilution and $50{ }^{\circ} \mathrm{C}$, the dissolution improved further to $13 \%$ 
and $5 \%$ undissolved solids for BY-102 and BY-106, which were the respective maximum dissolution levels observed during the current study. Dilution beyond $100 \%$ did not improve the dissolution of the B-106 waste.

Data are presented in Section 5 that explain the gross dissolution behavior in terms of solubilities of individual components in the waste. Evidence is provided there of the importance of double salts (sodium fluoride phosphate and sodium fluoride sulfate) in controlling the equilibrium concentrations of phosphate, fluoride, and sulfate in the dissolved waste solutions. Identification of solid phases in equilibrium with the ions in solution is important for computer modeling of the thermodynamics of the system.

The percentage of sodium in each tank that could be retrieved at reasonable dilution levels by pumping liquid from the tank (i.e., the percentage of total sodium that is in the liquid phase or can be dissolved) is estimated at $86 \%$ for BY-102, $98 \%$ for $\mathrm{BY}-106$, and $79 \%$ for $\mathrm{B}-106$.

For tanks BY-102 and BY-106, the solids that remain undissolved after two successive wash steps are mainly sodium salts, most of which have not been identified yet. Sodium oxalate is certainly a large component of these sparingly-soluble salts, but other unknown fluoride, phosphate, and sulfate salts are also major contributors. For tank B-106, the sludge components already discussed make up a large proportion of the undissolved solids. However, an unknown salt containing sodium and phosphate appears to be the largest single component of these solids.

Computer simulations of the saltcake series dissolution tests on tanks BY-102 and BY-106 were conducted by investigators at Mississippi State University, using ESP. Analytical results from the sequential dissolution tests were used as input to the program. The major trends in the experimental data were also seen in the simulation results. Quantitative predictions were obtained for concentrations of all major anions at dilutions of $100 \%$ and higher. Fluoride, phosphate, and sulfate concentrations showed marked discrepancies between the ESP predictions and the experimental data at dilutions below $100 \%$. Additional laboratory work is needed to determine the solid-liquid equilibria for the double salt systems, to provide better thermodynamic data for ESP to use in the predictions.

\subsection{COMPOSITION OF TANK WASTES}

\subsubsection{Tank BY-102}

Tank BY-102 is predicted by historical model (Agnew 1997) to contain $29 \mathrm{kgal}$ $(109 \mathrm{~kL})$ of sludge and $312 \mathrm{kgal}(1.2 \mathrm{ML})$ of saltcake, the saltcake having been deposited during the in-tank solidification (ITS) campaign of 1965-74. The saltcake waste type is identified by Agnew as type "BYSltCk". The current actual total waste volume (Sasaki 1997 ) in the tank is $277 \mathrm{kgal}(1.05 \mathrm{~kL})$. 
One eight-segment core sample (core 157) was taken from the tank in June, 1996. The first seven segments, plus a duplicate segment 5 (designated segment 5A) contained waste described as saltcake. Segment 1 was a partial segment. The bottom segment was approximately one-third full with liquid, and the remainder of the sampler was empty. Thus, none of the sludge layer was recovered.

Portions of segments 2 through 7 and $5 \mathrm{~A}$ were used to make the composite sample for this study. The chemical composition of the core sample and composite sample are shown in Table 8-1. The major components of the sample used for this study include (approximate weight percent): $\mathrm{Na}^{+}(28 \%), \mathrm{CO}_{3}{ }^{2-}(21 \%), \mathrm{NO}_{3}{ }^{-}(11 \%), \mathrm{Al}(\mathrm{OH})_{4}{ }^{-}$ $(6 \%), \mathrm{SO}_{4}{ }^{2-}(5 \%), \mathrm{PO}_{4}{ }^{3^{-}}(3 \%), \mathrm{C}_{2} \mathrm{O}_{4}{ }^{2^{-}}(2 \%), \mathrm{H}_{2} \mathrm{O}(26.5 \%)$. Sodium nitrate is a major salt in this waste, but it does not have the dominance that it does in other saltcakes, including BY-106.

\subsubsection{Tank BY-106}

Tank BY-106 is predicted by historical model to contain $44 \mathrm{kgal}$ (166 kL) of sludge and $544 \mathrm{kgal}(2.05 \mathrm{ML})$ of BYSltCk. The current actual total waste volume (Bell $1996)$ in the tank is $642 \mathrm{kgal}$ (2.43 ML).

One ten-segment core sample (core 121) was taken from the tank between December, 1995 and January, 1996. All ten segments contained waste described as saltcake. Segment 1 was a partial segment. Portions of segments 3 through 8 were used to make the composite sample for this study. The chemical composition of the core sample and composite sample are shown in Table 8-2. The major components of the sample used for this study include (approximate weight percent): $\mathrm{NO}_{3}{ }^{-}(41 \%), \mathrm{Na}^{+}$ $(25 \%), \mathrm{Al}(\mathrm{OH})_{4}{ }^{-}(8 \%), \mathrm{CO}_{3}{ }^{2-}(7 \%), \mathrm{NO}_{2}{ }^{-}(3 \%), \mathrm{SO}_{4}{ }^{2^{-}}(1 \%), \mathrm{C}_{2} \mathrm{O}_{4}{ }^{2-}$ (1\%), $\mathrm{H}_{2} \mathrm{O}(15 \%)$. Sodium nitrate is definitely the dominant salt in this waste.

\subsubsection{Tank B-106}

Tank B-106 is predicted by historical model to contain $116 \mathrm{kgal}$ ( $438 \mathrm{~kL}$ ) of saltcake derived from the 1951-53 operation of the 242-B evaporator. This waste type is defined by Agnew as type "BSltCk". The current actual total waste volume (McCain $1996)$ in the tank is $117 \mathrm{kgal}(443 \mathrm{~kL})$.

Two core samples (cores 93 and 94), two segments each, were taken from the tank in July, 1995. In both cores, the upper segment and the top half of the lower segment were said to contain light-to-dark brown sludge. The bottom half of the bottom segment contained light green sludge. Portions of segments $1 U$ (upper half of segment 1), IL (lower half of segment 1), and $2 U$ were used to make the composite sample for this study. 
HNF-3437, Rev. 0

The chemical composition of the core sample and composite sample are shown in Table 8-3. The major components of the composite sample used in this study include (approximate weight percent): $\mathrm{NO}_{3}^{-}(16 \%), \mathrm{Na}^{+}(9.5 \%), \mathrm{NO}_{2}{ }^{-}(1 \%), \mathrm{SO}_{4}{ }^{2-}(1 \%), \mathrm{Fe}$ $(1 \%), \mathrm{U}(1 \%), \mathrm{H}_{2} \mathrm{O}(58 \%)$. This waste had a much higher percentage of water and of water-insoluble components $(\mathrm{Al}, \mathrm{Bi}, \mathrm{Ca}, \mathrm{Fe}, \mathrm{U})$ than the other two tank waste samples. 
HNF-3437, Rev. 0

\subsection{DESCRIPTION OF LABORATORY STUDIES}

Composite samples of actual tank waste were prepared in a hotcell. Smaller samples were handled in fume hoods. The procedures, data, and observations are recorded in controlled laboratory notebook HNF-N-70-2. The procedures are described in this section, and the raw data are shown in the following section (3.0).

All dissolution tests were done on composite samples made by combining representative core segment samples from each tank. Two types of dissolution tests were done. The sequential dissolution tests were done to establish an overall saltcake waste composition for each tank, as well as to define basic dissolution behavior. The series dissolution tests were done to establish dissolution characteristics of individual salts as a function of the amount of diluent added. Both types of tests are described in detail in sections 2.2 and 2.3 .

\subsection{PREPARATION OF COMPOSITE SAMPLES}

Composite samples were made by combining archived fractions of individual core segment samples into a single composite jar for each tank. The entire contents of each archive jar were transferred to the composite jar. The waste in the completed composite jar was mixed thoroughly before transferring an aliquot from the hotcell to the fume hood. Weights of individual core segment samples used for each composite sample are shown in Table 2-1. Aliquots of each composite sample were analyzed for percent water by oven-drying at $120^{\circ} \mathrm{C}$ and by thermogravimetric analysis (TGA).

Comparisons between analytical data for the composite samples and the tank core samples as reported in the Tank Characterization Report (TCR) for each tank are discussed in Section 8.

\subsection{SEQUENTIAL DISSOLUTION TESTS}

The following tests were performed on composite samples from all three tanks BY-102, BY-106, and B-106.

Approximately $30 \mathrm{~g}$ of tank composite sample were transferred into each of two $50 \mathrm{~mL}$ centrifuge cones, and an equal weight of water was added. [For tank B-106, two additional cones were treated with $3 \mathrm{M} \mathrm{NaOH}$ instead of water.] The cones were mixed with a vortex mixer, then placed on a rocker and tumbled end-over-end for at least 24 hours at ambient temperature (approximately $23^{\circ} \mathrm{C}$ ). The cones were then centrifuged for one hour at approximately $2000 \mathrm{rpm}$. 
HNF-3437, Rev. 0

Table 2-1. Segment Samples Used to Create Core Composite Samples

\begin{tabular}{|c|c|c|c|c|}
\hline Tank & Core & Segment ${ }^{a}$ & Jar \# & Weight, g \\
\hline \multirow{12}{*}{ BY-102 } & 157 & 2 & 10668 & 80.93 \\
\hline & 157 & 3 & 10669 & 57.18 \\
\hline & 157 & 4 & 10845 & 62.64 \\
\hline & 157 & 5 & 10961 & 58.85 \\
\hline & 157 & $5 A$ & 10846 & 35.17 \\
\hline & 157 & 6 & 10670 & 81.99 \\
\hline & 157 & 6 & 10671 & 34.14 \\
\hline & 157 & 7 & 10780 & 33.05 \\
\hline & 157 & 7 & 10781 & 42.78 \\
\hline & 157 & 7 & 10782 & 12.96 \\
\hline & 157 & 7 & 10847 & 62.06 \\
\hline & & Composite & Total $\mathrm{Wt}=$ & 561.75 \\
\hline \multirow{7}{*}{ BY-106 } & 121 & 3 & 8531 & 46.31 \\
\hline & 121 & 4 & 8533 & 61.11 \\
\hline & 121 & 5 & 8536 & 83.17 \\
\hline & 121 & 6 & 8541 & 129.35 \\
\hline & 121 & 7 & 8544 & 64.15 \\
\hline & 121 & 8 & 8552 & 68.55 \\
\hline & & Composite & Total Wt $=$ & 449.94 \\
\hline \multirow{8}{*}{ B-106 } & 93 & $2 \mathrm{U}$ & 7416 & 31.64 \\
\hline & 93 & $1 \mathrm{~L}$ & 7420 & 55.52 \\
\hline & 94 & $\mathrm{IU}$ & 7423 & 46.22 \\
\hline & 94 & $2 U$ & 7425 & 52.09 \\
\hline & 94 & $1 \mathrm{~L}$ & 8454 & 22.05 \\
\hline & 94 & $1 \mathrm{U}$ & 9687 & 50.64 \\
\hline & 94 & $2 U$ & 9688 & 4.98 \\
\hline & & Composite & Total $\mathrm{Wt}=$ & 263.14 \\
\hline
\end{tabular}

a Segment $\mathrm{l}$ is at top of waste; $\mathrm{U}=$ upper half of segment; $\mathrm{L}=$ lower half of segment.

The supernatant liquids from both cones were combined into a sample bottle. The liquid was analyzed for metals by inductively coupled plasma spectroscopy (ICP), anions by ion chromatography (IC), total inorganic carbon (TIC), total organic carbon (TOC), free hydroxide $\left(\mathrm{OH}^{-}\right)$, density, percent water by oven drying at $120^{\circ} \mathrm{C}$, and radionuclides by gamma energy analysis (GEA).

The weight and volume of centrifuged solids in each cone were recorded. Then $30 \mathrm{~g}$ of water (or $3 \mathrm{M} \mathrm{NaOH}$ for the appropriate $\mathrm{B}-106$ cones) were added to each cone. The cones were mixed again with a vortex mixer, then tumbled end-over-end for at least 24 hours at ambient temperature. The cones were centrifuged again, and the supernatant 
liquids combined as before in a new sample bottle. The second wash solution was analyzed for the same components as the first wash solution.

The volume and weight of undissolved centrifuged solids were recorded. Then the solids were transferred from the cones into a sample vial, again combining the solids from both cones into a single sample vial. The solids were analyzed for TIC, TOC, and $\% \mathrm{H}_{2} \mathrm{O}$ by TGA. The solids were also subjected to acid digestion, and the resulting solution was analyzed for metals by ICP.

\subsection{SERIES DISSOLUTION TESTS}

The following tests were performed on composite samples from two of the three tanks - BY-102 and BY-106.

Four to six grams of composite sample were placed into each of six $15 \mathrm{~mL}$ centrifuge cones. An amount of water was added to each cone representing 50, 100, 150, 200,250 , or $300 \%$ by weight of the composite sample in the cone. Each cone was mixed with a vortex mixer, then tumbled end-over-end for at least 24 hours at ambient temperature. The cones were centrifuged for 30 minutes at approximately $2000 \mathrm{rpm}$. The total volume and centrifuged solids volume in each cone were recorded. The liquid from each cone was decanted into a sample vial to be analyzed for density, TIC, and IC. The cones were re-weighed to determine the weight of undissolved centrifuged solids.

The series dissolution test just described was repeated, except that the 24-hour tumbling was done in an oven maintained at $50^{\circ} \mathrm{C}$. The decanted supernatant liquid samples were held at ambient temperature for 24 hours to watch for signs of precipitation that might be caused by cooling. If solids formed, the samples were diluted $1: 1$ by weight with water to re-dissolve the crystals before the samples were analyzed. This dilution was required for all of the BY-102 samples and the 50\% sample from BY-106. 


\subsection{RESULTS - RAW DATA}

"Raw data" are values obtained for net weight and volume measurements and chemical analyses. They are reproduced here for documentation purposes. Sample-tosample comparisons should not be made on raw data measurements because of differences in initial sample weights and volumes. Comparisons must be based on normalized data, accounting for initial volume and weight differences. The normalized data are presented in Section 4.

\subsection{Tank BY-102}

The aliquot of the composite sample taken for $\% \mathrm{H}_{2} \mathrm{O}$ analysis was labeled 2BYTC, and was assigned Labcore number S98R000195. (Labcore is the laboratory information and management system used at the 222-S Laboratory.) Analytical results were $26.53 \% \mathrm{H}_{2} \mathrm{O}$ by TGA and $26.0 \%$ by oven-drying.

\subsubsection{BY-102 Sequential Dissolution Test}

Two $50 \mathrm{~mL}$ centrifuge cones were labeled SEQA and SEQB. The procedure was described in Section 2.2. Weights, volumes, and analytical sample identification numbers of the various fractions are shown in Table 3-1. Sample analytical results are shown in Table $3-2$.

\subsubsection{BY-102 Series Dissolution Test, Ambient Temperature}

Six $15 \mathrm{~mL}$ centrifuge cones were labeled BY2-A through BY2-F. The procedure was described in Section 2.3. Weights, volumes, and analytical sample identification numbers of the various fractions are shown in Table 3-3. Sample analytical results are shown in Table $3-4$.

\subsubsection{BY-102 Series Dissolution Test, $50^{\circ} \mathrm{C}$}

Six $15 \mathrm{~mL}$ centrifuge cones were labeled BY2H-A through BY2H-F. The procedure was described in Section 2.3. Weights, volumes, and analytical sample identification numbers of the various fractions are shown in Table 3-5. Liquid samples. were diluted to dissolve crystals that formed when the samples cooled from $50^{\circ} \mathrm{C}$ to ambient temperature. Sample analytical results for the diluted liquid samples are shown in Table 3-6a. The corresponding undiluted sample results are shown in Table 3-6b. 
Table 3-1. Raw Data, Tank BY-102 Sequential Dissolution Test. (weights in grams, volumes in $\mathrm{mL}$ )

\begin{tabular}{|c|c|c|}
\hline & SEQA & SEQB \\
\hline Sample Wt & 30.670 & 32.183 \\
\hline $\mathrm{Wt} \mathrm{H}_{2} \mathrm{O}$ Added ( $1^{\text {st }}$ wash) & 29.310 & 30.278 \\
\hline Total Vol & 44.0 & 45.0 \\
\hline $1^{\text {st }}$ Water Wash Sample ID ${ }^{\mathrm{a}}$ & \multicolumn{2}{|c|}{ 2BYSEQAB1/ S98R000204 } \\
\hline $\mathrm{CSOl}^{\mathrm{b}} \mathrm{Vol}$ & 15.0 & 4.0 \\
\hline CSol Wt & 22.995 & 16.222 \\
\hline Wt $\mathrm{H}_{2} \mathrm{O}$ Added ( $2^{\text {nd }}$ wash) & 30.171 & 29.160 \\
\hline Total Vol & 44.5 & 38.0 \\
\hline $2^{\text {nd }}$ Water Wash Sample ID & \multicolumn{2}{|c|}{ 2BYSEQAB2 / S98R000206 } \\
\hline $\mathrm{CSol} \mathrm{Vol}$ & 6.0 & 6.0 \\
\hline CSol Wt & 8.628 & 8.868 \\
\hline CSol Sample ID & \multicolumn{2}{|c|}{ 2BYSEQABS / S 98 R000207 and 208} \\
\hline
\end{tabular}

a decanted supernatant liquid; customer ID number / Labcore ID number

${ }^{\mathrm{b}} \mathrm{CSOl}=$ centrifuged solids 
Table 3-2. Analytical Results, Tank BY-102 Sequential Dissolution Test.

\begin{tabular}{|c|c|c|c|}
\hline Analyte & $\begin{array}{c}1^{\text {st }} \text { Water Wash } \\
\text { 2BYSEQABl } \\
\text { S98R000204 } \\
(\mu \mathrm{g} / \mathrm{mL})\end{array}$ & $\begin{array}{c}2^{\text {nd }} \text { Water Wash } \\
\text { 2BYSEQAB2 } \\
\text { S98R000206 } \\
(\mu \mathrm{g} / \mathrm{mL})\end{array}$ & $\begin{array}{c}\text { CSol } \\
\text { 2BYSEQABS } \\
\text { S98R000207-8 } \\
(\mu g / g)\end{array}$ \\
\hline $\mathrm{Al}$ & 8710 & 1280 & 21700 \\
\hline $\mathrm{Ca}$ & & & 1540 \\
\hline $\mathrm{Cr}$ & 1110 & 168 & 2350 \\
\hline $\mathrm{Fe}$ & & & 1910 \\
\hline $\mathrm{K}$ & 1320 & $<300$ & $<970$ \\
\hline $\mathrm{Mg}$ & & & 366 \\
\hline $\mathrm{Mn}$ & & . & 876 \\
\hline $\mathrm{Na}$ & 156000 & 70000 & 136000 \\
\hline $\mathrm{Ni}$ & & & 951 \\
\hline $\mathrm{P}$ & 604 & 1150 & 29000 \\
\hline $\mathrm{Pb}$ & & & 254 \\
\hline $\mathrm{S}$ & 3590 & 13500 & 5390 \\
\hline Si & 257 & 140 & 1080 \\
\hline $\mathrm{U}$ & & & 2290 \\
\hline $\mathrm{F}^{-}$ & 2690 & 7940 & $\mathrm{na}^{\mathrm{a}}$ \\
\hline $\mathrm{Cl}^{-}$ & 1060 & $<173$ & $\mathrm{na}$ \\
\hline $\mathrm{NO}_{2}$ & 14800 & 2870 & na \\
\hline $\mathrm{NO}_{3}^{-}$ & 92300 & 12300 & na \\
\hline $\mathrm{PO}_{4}{ }^{3+}$ & 1680 & 3810 & na \\
\hline $\mathrm{SO}_{4}{ }^{2+}$ & 10500 & 36000 & na \\
\hline $\mathrm{C}_{2} \mathrm{O}_{4}{ }^{2-}$ & 930 & 3860 & na \\
\hline TIC & $(32700)^{b}$ & 5890 & 3140 \\
\hline TOC & 1020 & 1110 & 14300 \\
\hline $\mathrm{OH}^{-}$ & 9020 & 1400 & na \\
\hline${ }^{137} \mathrm{Cs}(\mu \mathrm{Ci} / \mathrm{mL})$ & 36.1 & 6.3 & na \\
\hline Density $(\mathrm{g} / \mathrm{mL})$ & 1.232 & 1.125 & na \\
\hline$\% \mathrm{H}_{2} \mathrm{O}$ & 69.0 & 84.0 & 56.97 \\
\hline
\end{tabular}

${ }^{a}$ na $=$ not analyzed

${ }^{\mathrm{b}}$ parentheses denote unrealistic analytical result 
Table 3-3. Raw Data, Tank BY-102 Series Dissolution Test, Ambient Temperature (weights in grams, volumes in $\mathrm{mL}$ )

\begin{tabular}{|c|c|c|c|c|c|c|}
\hline Wt\% Dilution: & $51 \%$ & $100 \%$ & $150 \%$ & $200 \%$ & $251 \%$ & $299 \%$ \\
\hline Cone ID: & BY2-E & BY2-B & BY2-C & BY2-D & BY2-F & BY2-A \\
\hline Sample Wt & 4.785 & 4.338 & 4.685 & 4.662 & 4.538 & 3.600 \\
\hline Wt H2O Added & 2.447 & 4.345 & 7.040 & 9.325 & 11.382 & 10.767 \\
\hline Total Vol & 4.6 & 6.3 & 9.2 & 11.5 & 13.5 & 12.2 \\
\hline CSol Vol & 3.1 & 2.0 & 1.5 & 1.0 & 0.8 & 0.6 \\
\hline CSol Wt & 5.123 & 3.274 & 2.008 & 1.548 & 1.344 & 0.962 \\
\hline Supernate ID & $2 \mathrm{BYE} 1$ & $2 \mathrm{BYBl}$ & $2 \mathrm{BYCl}$ & $2 \mathrm{BYD}$ & $2 \mathrm{BYF} 1$ & $2 \mathrm{BYAl}$ \\
\hline S98R000 ... & 201 & 197 & 198 & 200 & 202 & 196 \\
\hline
\end{tabular}

Table 3-4. Analytical Results, Tank BY-102 Series Dissolution Test, Ambient Temp. ( $\mu \mathrm{g} / \mathrm{mL}$ except as noted)

\begin{tabular}{|c|c|c|c|c|c|c|}
\hline Wt $\%$ Dilution: & $51 \%$ & $100 \%$ & $150 \%$ & $200 \%$ & $251 \%$ & $299 \%$ \\
\hline Supernate ID & $2 \mathrm{BYE} 1$ & $2 \mathrm{BYB} 1$ & $2 \mathrm{BYCl}$ & $2 \mathrm{BYD} 1$ & $2 \mathrm{BYF} 1$ & $2 \mathrm{BYA1}$ \\
\hline $\mathrm{S} 98 \mathrm{R} 000 \ldots$ & 201 & 197 & 198 & 200 & 202 & 196 \\
\hline Density $(\mathrm{g} / \mathrm{mL})$ & 1.368 & 1.267 & 1.230 & 1.203 & 1.190 & 1.166 \\
\hline $\mathrm{TIC}$ & 17600 & 16200 & 16300 & 13000 & 11000 & 9210 \\
\hline $\mathrm{F}$ & 999 & 2780 & 4150 & 5530 & 5060 & 4460 \\
\hline $\mathrm{Cl}^{-}$ & 1840 & 1010 & 583 & 464 & 398 & 390 \\
\hline $\mathrm{NO}_{2} \mathrm{NO}_{3}$ & 25200 & 13700 & 8520 & 7550 & 6180 & 4880 \\
\hline $\mathrm{PO}_{4}{ }^{3-}$ & 169000 & 87700 & 54500 & 40300 & 35800 & 29500 \\
\hline $\mathrm{SO}_{4}{ }^{2-}$ & 1310 & 1980 & 2430 & 2410 & 3130 & 3700 \\
\hline $\mathrm{C}_{2} \mathrm{O}_{4}{ }^{2-}$ & 5520 & 12600 & 19300 & 22700 & 20000 & 17200 \\
\hline
\end{tabular}


HNF-3437, Rev. 0

Table 3-5. Raw Data, Tank BY-102 Series Dissolution Test, $50^{\circ} \mathrm{C}$ (weights in grams, volumes in $\mathrm{mL}$ )

\begin{tabular}{|l|c|c|c|c|c|c|}
\hline Wt\% Dilution: & $50 \%$ & $99 \%$ & $148 \%$ & $198 \%$ & $249 \%$ & $297 \%$ \\
\hline \multicolumn{1}{|c|}{ Cone ID: } & BY2H-A & BY2H-B & BY2H-D & BY2H-E & BY2H-F & BY2H-C \\
\hline Sample Wt & 6.010 & 6.160 & 6.348 & 4.276 & 4.386 & 3.782 \\
\hline Wt H2O Added & 3.000 & 6.112 & 9.422 & 8.462 & 10.903 & 11.232 \\
\hline Total Vol & 5.8 & 8.2 & 11.8 & $7.5^{\mathrm{a}}$ & $7.0^{\mathrm{a}}$ & 12.5 \\
\hline CSol Vol & 2.2 & 1.8 & 1.2 & $0.4^{\mathrm{a}}$ & $0.3^{\mathrm{a}}$ & 0.2 \\
\hline CSol Wt & 4.250 & 2.937 & 2.083 & $0.939^{\mathrm{a}}$ & $0.583^{\mathrm{a}}$ & 0.502 \\
\hline Supernate ID & BY2HAL & BY2HBL & BY2HDL & BY2HEL & BY2HFL & BY2HCL \\
\hline S98R000 .. & 210 & 211 & 214 & 215 & 216 & 212 \\
\hline Sup Sample Wt & 2.574 & 7.008 & 11.754 & 6.467 & 6.508 & 12.893 \\
\hline Sup H B Added $_{2.559}$ & 6.907 & 11.597 & 6.619 & 6.535 & 11.670 \\
\hline Density Diluted & 1.172 & 1.138 & 1.112 & 1.086 & 1.062 & 1.069 \\
\hline Vol Diluted & 4.38 & 12.23 & 21.00 & 12.05 & 12.28 & 22.98 \\
\hline Vol Undiluted & 1.82 & 5.32 & 9.40 & 5.43 & 5.75 & 11.31 \\
\hline Density Undil & 1.41 & 1.32 & 1.25 & 1.19 & 1.13 & 1.14 \\
\hline Diln Factor (v/v) & 2.41 & 2.30 & 2.23 & 2.22 & 2.14 & 2.03 \\
\hline
\end{tabular}

${ }^{a}$ Cones $\mathrm{E}$ and $\mathrm{F}$ broke during heating, and some sample was lost.

Table 3-6a. Analytical Results, Tank BY-102 Series Dissolution Test, $50^{\circ} \mathrm{C}$ (diluted samples; $\mu \mathrm{g} / \mathrm{mL}$ except as noted)

\begin{tabular}{|c|c|c|c|c|c|c|}
\hline Wt $\%$ Dilution: & $50 \%$ & $99 \%$ & $148 \%$ & $198 \%$ & $249 \%$ & $297 \%$ \\
\hline Supernate ID & BY2HAL & BY2HBL & BY2HDL & BY2HEL & BY2HFL & BY2HCL \\
\hline S98R000 $\ldots$ & 210 & 211 & 214 & 215 & 216 & 212 \\
\hline Density $(\mathrm{g} / \mathrm{mL})$ & 1.172 & 1.138 & 1.112 & 1.086 & 1.062 & 1.069 \\
\hline $\mathrm{TIC}$ & 10400 & 10100 & 7650 & 6040 & 5010 & 4250 \\
\hline $\mathrm{F}^{-}$ & 880 & 1270 & 1740 & 2540 & 2570 & 1820 \\
\hline $\mathrm{Cl}^{-}$ & 518 & 276 & 214 & 222 & 186 & $<88$ \\
\hline $\mathrm{NO}_{2}{ }^{-}$ & 8800 & 5330 & 3970 & 3130 & 2610 & 2300 \\
\hline $\mathrm{NO}_{3} \mathrm{PO}_{4}{ }^{-}$ & 55200 & 34300 & 24500 & 21100 & 15900 & 12500 \\
\hline $\mathrm{SO}_{4}{ }^{2-}$ & 3450 & 4320 & 3620 & 5320 & 5120 & $(1980)$ \\
\hline $\mathrm{C}_{2} \mathrm{O}_{4}{ }^{2-}$ & 1600 & 3920 & 6150 & 8980 & 9250 & $(5850)$ \\
\hline
\end{tabular}


Table 3-6b. Analytical Results, Tank BY-102 Series Dissolution Test, $50^{\circ} \mathrm{C}$ (undiluted samples; $\mu \mathrm{g} / \mathrm{mL}$ except as noted)

\begin{tabular}{|c|c|c|c|c|c|c|}
\hline Wt\% Dilution: & $50 \%$ & $99 \%$ & $148 \%$ & $198 \%$ & $249 \%$ & $297 \%$ \\
\hline Supernate ID & BY2HAL & BY2HBL & BY2HDL & BY2HEL & BY2HFL & BY2HCL \\
\hline S98R000 $\ldots$ & 210 & 211 & 214 & 215 & 216 & 212 \\
\hline Density $(g / m L)$ & 1.41 & 1.32 & 1.25 & 1.19 & 1.13 & 1.14 \\
\hline $\mathrm{TIC}$ & 25000 & 23200 & 17100 & 13400 & 10700 & 8640 \\
\hline $\mathrm{F}$ & 2120 & 2920 & 3890 & 5640 & 5490 & 3700 \\
\hline $\mathrm{Cl}$ & 1250 & 634 & 478 & 493 & 398 & $<176$ \\
\hline $\mathrm{NO}_{2}{ }^{-}$ & 21200 & 12200 & 8870 & 6940 & 5580 & 4670 \\
\hline $\mathrm{NO}_{3}$ & 133000 & 78800 & 54700 & 46800 & 34000 & 25400 \\
\hline $\mathrm{PO}_{4}{ }^{2-}$ & 8300 & 9930 & 8080 & 11800 & 10900 & $(4020)$ \\
\hline $\mathrm{SO}_{4}{ }^{-2}$ & 3850 & 9010 & 13700 & 19900 & 19800 & $(11900)$ \\
\hline $\mathrm{C}_{2} \mathrm{O}_{4}{ }^{2}$ & $<1200$ & 1760 & 2280 & 2750 & 3210 & 3250 \\
\hline
\end{tabular}

\subsection{Tank BY-106}

Two aliquots of the composite sample taken for $\% \mathrm{H}_{2} \mathrm{O}$ analysis were labeled BY106CMPD (Labcore S98R000001) and BY6COMPRR2 (Labcore S98R000029). The analytical result for the first sample was $14.05 \% \mathrm{H}_{2} \mathrm{O}$ by TGA. Results for the second aliquot were $14.68 \% \mathrm{H}_{2} \mathrm{O}$ by TGA and $14.7 \% \mathrm{H}_{2} \mathrm{O}$ by oven-drying. The results from the second sample are used in the calculations in Section 4.

\subsubsection{BY-106 Sequential Dissolution Test}

Two $50 \mathrm{~mL}$ centrifuge cones were labeled BY6-SEQA and BY6-SEQB. The procedure was described in Section 2.2. Weights, volumes, and analytical sample identification numbers of the various fractions are shown in Table 3-7. Sample analytical results are shown in Table $3-8$.

\subsubsection{BY-106 Series Dissolution Test, Ambient Temperature}

Six $15 \mathrm{~mL}$ centrifuge cones were labeled $\mathrm{A}$ through $\mathrm{F}$. The procedure was described in Section 2.3. Weights, volumes, and analytical sample identification numbers of the various fractions are shown in Table 3-9. Sample analytical results are shown in Table $3-10$. 


\subsubsection{BY-106 Series Dissolution Test, $50^{\circ} \mathrm{C}$}

Six $15 \mathrm{~mL}$ centrifuge cones were labeled BY6H-A through BY6H-F. The procedure was described in Section 2.3. Weights, volumes, and analytical sample identification numbers of the various fractions are shown in Table 3-11. The liquid sample from cone BY6H-A was diluted to dissolve crystals that formed when the sample cooled from $50^{\circ} \mathrm{C}$ to ambient temperature. Sample analytical results for the undiluted liquid sample (dilution-corrected) are shown in Table 3-12 with the results for the other five samples which were not diluted.

Table 3-7. Raw Data, Tank BY-106 Sequential Dissolution Test. (weights in grams, volumes in $\mathrm{mL}$ )

\begin{tabular}{|c|c|c|}
\hline & BY6-SEQA & BY6-SEQB \\
\hline Sample Wt & 30.017 & 30.091 \\
\hline Wt $\mathrm{H}_{2} \mathrm{O}$ Added ( $1^{\text {st }}$ wash $)$ & 30.026 & 30.139 \\
\hline Total Vol & 45.2 & 46.7 \\
\hline $1^{\text {st }}$ Water Wash Sample ID & \multicolumn{2}{|c|}{ BY6SEQAB1/ S98R000024 } \\
\hline $\mathrm{CSOl}^{\mathrm{b}} \mathrm{Vol}$ & 4.9 & 4.8 \\
\hline $\mathrm{CSOl} \mathrm{Wt}$ & 6.702 & 6.225 \\
\hline $\mathrm{Wt} \mathrm{H}_{2} \mathrm{O}$ Added ( $2^{\text {nd }}$ wash $)$ & 29.440 & 31.020 \\
\hline Total Vol & 34.0 & 35.5 \\
\hline $2^{\text {nd }}$ Water Wash Sample ID & \multicolumn{2}{|c|}{ BY6SEQAB2/ S98R000025 } \\
\hline CSol Vol & 3 & 3 \\
\hline CSol Wt & 1.570 & 1.253 \\
\hline CSol Sample ID & \multicolumn{2}{|c|}{ BY6SEQABCS / S98R00027 and 28} \\
\hline
\end{tabular}

a decanted supernatant liquid; customer ID number / Labcore ID number

${ }^{\mathrm{b}} \mathrm{CSol}=$ centrifuged solids 
Table 3-8. Analytical Results, Tank BY-106 Sequential Dissolution Test.

\begin{tabular}{|c|c|c|c|}
\hline Analyte & $\begin{array}{c}1^{\text {st }} \text { Water Wash } \\
\text { BY6SEQAB 1 } \\
\text { S98R000024 } \\
(\mu \mathrm{g} / \mathrm{mL})\end{array}$ & $\begin{array}{c}2^{\text {nd }} \text { Water Wash } \\
\text { BY6SEQAB2 } \\
\text { S98R000025 } \\
(\mu \mathrm{g} / \mathrm{mL})\end{array}$ & $\begin{array}{c}\text { CSol } \\
\text { BY6SEQABCD } \\
\text { S98R000027-28 } \\
(\mu \mathrm{g} / \mathrm{g})\end{array}$ \\
\hline $\mathrm{Al}$ & 10300 & 1110 & 13000 \\
\hline $\mathrm{Ca}$ & $<40$ & $<10$ & 2770 \\
\hline $\mathrm{Cr}$ & 245 & 34 & 16200 \\
\hline$\overline{\mathrm{Fe}}$ & $<20$ & $<5$ & 4780 \\
\hline $\mathrm{K}$ & 1660 & 158 & $<200$ \\
\hline $\mathrm{Mg}$ & & & 214 \\
\hline $\mathrm{Mn}$ & & & 248 \\
\hline $\mathrm{Na}$ & 157000 & 29400 & 85100 \\
\hline $\mathrm{Ni}$ & $<8$ & $<2$ & 1230 \\
\hline $\mathrm{P}$ & 239 & 396 & 742 \\
\hline $\mathrm{Pb}$ & $<40$ & $<10$ & 753 \\
\hline $\mathrm{S}$ & 938 & 2910 & 2330 \\
\hline $\mathrm{Si}$ & 85 & 34 & 340 \\
\hline $\mathrm{Sr}$ & $<40$ & $<1$ & 1330 \\
\hline $\mathrm{U}$ & $<200$ & $<50$ & 4120 \\
\hline $\mathrm{F}^{-}$ & 2085 & 3130 & $n a^{a}$ \\
\hline $\mathrm{Cl}^{-}$ & 1100 & 118 & na \\
\hline $\mathrm{NO}_{2}$ & 18200 & 2090 & na \\
\hline $\mathrm{NO}_{3}{ }^{-1}$ & 273000 & 30450 & na \\
\hline $\mathrm{PO}_{4}^{3-}$ & 1500 & 1290 & na \\
\hline $\mathrm{SO}_{4}{ }^{2-}$ & 1710 & 8800 & na \\
\hline $\mathrm{C}_{2} \mathrm{O}_{4}{ }^{-2}$ & 475 & 7400 & na \\
\hline TIC & 9590 & 1060 & 1340 \\
\hline TOC & 952 & 1970 & 29200 \\
\hline $\mathrm{OH}$ & 7660 & 881 & na \\
\hline${ }^{137} \mathrm{Cs}(\mu \mathrm{Ci} / \mathrm{mL})$ & 52.4 & 6.3 & na \\
\hline Density $(\mathrm{g} / \mathrm{mL})$ & 1.336 & 1.087 & na \\
\hline$\% \mathrm{H}_{2} \mathrm{O}$ & 58.2 & 91.4 & 69.91 \\
\hline
\end{tabular}

${ }^{a}$ na $=$ not analyzed 
Table 3-9. Raw Data, Tank BY-106 Series Dissolution Test, Ambient Temperature (weights in grams, volumes in $\mathrm{mL}$ )

\begin{tabular}{|l|c|c|c|c|c|c|}
\hline Wt $\%$ Dilution: & $46 \%$ & $99 \%$ & $164 \%$ & $204 \%$ & $246 \%$ & $292 \%$ \\
\hline Cone ID: & $\mathrm{A}$ & $\mathrm{B}$ & $\mathrm{C}$ & $\mathrm{D}$ & $\mathrm{E}$ & $\mathrm{F}$ \\
\hline Sample Wt & 4.637 & 6.352 & 4.030 & 3.961 & 4.446 & 4.187 \\
\hline Wt H2O Added & 2.143 & 6.313 & 6.599 & 8.078 & 10.928 & 12.224 \\
\hline Total Vol & 4.6 & 8.9 & 8.5 & 9.7 & 12.9 & 13.9 \\
\hline CSol Vol & 1.9 & 1.0 & 0.4 & 0.4 & 0.3 & 0.2 \\
\hline CSol Wt & 3.245 & 1.716 & 0.828 & 0.767 & 0.694 & 0.666 \\
\hline Supernate ID & $\mathrm{AX} 1 \mathrm{DH}$ & $\mathrm{BX} 1 \mathrm{DH}$ & $\mathrm{CX} 1 \mathrm{DH}$ & $\mathrm{DX} 2 \mathrm{DH}$ & $\mathrm{EX} 2 \mathrm{DH}$ & $\mathrm{FX} 2 \mathrm{DH}$ \\
\hline S98R000... & 002 & 004 & 005 & 006 & 008 & 009 \\
\hline
\end{tabular}

Table 3-10. Analytical Results, Tank BY-106 Series Dissolution Test, Ambient Temp. ( $\mu \mathrm{g} / \mathrm{mL}$ except as noted)

\begin{tabular}{|c|c|c|c|c|c|c|}
\hline Wt\% Dilution: & $46 \%$ & $99 \%$ & $164 \%$ & $204 \%$ & $246 \%$ & $292 \%$ \\
\hline Supernate ID & $\mathrm{AX} 1 \mathrm{DH}$ & $\mathrm{BX} 1 \mathrm{DH}$ & $\mathrm{CX} 1 \mathrm{DH}$ & $\mathrm{DX} 2 \mathrm{DH}$ & $\mathrm{EX} 2 \mathrm{DH}$ & $\mathrm{FX} 2 \mathrm{DH}$ \\
\hline $\mathrm{S} 98 \mathrm{R} 000 \ldots$ & 002 & 004 & 005 & 006 & 008 & 009 \\
\hline Density $(\mathrm{g} / \mathrm{mL})$ & 1.400 & 1.353 & 1.260 & 1.233 & 1.198 & 1.191 \\
\hline $\mathrm{TIC}$ & 14200 & 8180 & 5850 & 5480 & 4310 & 3930 \\
\hline $\mathrm{F}$ & 1250 & 2085 & 3020 & 2650 & 2245 & 1850 \\
\hline $\mathrm{Cl}^{-}$ & 1490 & 1154 & 774 & 626 & 580 & 470 \\
\hline $\mathrm{NO}_{2}{ }^{-}$ & 30900 & 20600 & 14300 & 11100 & 9940 & 8550 \\
\hline $\mathrm{NO}_{3}{ }^{-2}$ & 269000 & 296000 & 198000 & 152000 & 129000 & 110000 \\
\hline $\mathrm{PO}_{4}{ }^{3-}$ & 778 & 791 & 870 & 900 & 1130 & 554 \\
\hline $\mathrm{SO}_{4}{ }^{2-}$ & 1230 & 2220 & 5345 & 4620 & 3960 & 3430 \\
\hline $\mathrm{C}_{2} \mathrm{O}_{4}{ }^{2-}$ & $<541$ & 475 & 1010 & 1400 & 1785 & 2210 \\
\hline
\end{tabular}


Table 3-11. Raw Data, Tank BY-106 Series Dissolution Test, $50^{\circ} \mathrm{C}$ (weights in grams, volumes in $\mathrm{mL}$ )

\begin{tabular}{|c|c|c|c|c|c|c|}
\hline Wt $\%$ Dilution: & $49 \%$ & $99 \%$ & $151 \%$ & $191 \%$ & $249 \%$ & $295 \%$ \\
\hline Cone ID: & $\mathrm{BY} 6 \mathrm{H}-\mathrm{A}$ & BY6H-B & $\mathrm{BY} 6 \mathrm{H}-\mathrm{C}$ & BY6H-D & BY6H-E & BY6H-F \\
\hline Sample Wt & 7.773 & 7.260 & 4.272 & 4.775 & 3.900 & 4.119 \\
\hline Wt H2O Added & 3.779 & 7.181 & 6.445 & 9.119 & 9.706 & 12.167 \\
\hline Initial Total Wt & 11.552 & 14.441 & 10.717 & 13.894 & 13.606 & 16.286 \\
\hline Final Total $\mathrm{Wt}^{\mathrm{a}}$ & 11.182 & 10.454 & 10.511 & 8.838 & 11.629 & 15.800 \\
\hline Final Total Vol & 7.1 & 7.8 & 8.3 & 7.1 & 9.6 & 13.5 \\
\hline CSol Vol & 1.9 & 0.9 & 0.25 & 0.2 & 0.2 & 0.15 \\
\hline CSol Wt & 3.849 & 1.387 & 0.484 & 0.473 & 0.253 & 0.228 \\
\hline Supernate ID & $\mathrm{AHL}$ & $\mathrm{BHL}$ & $\mathrm{CHL}$ & DHI & EHL & FHL \\
\hline S98R000... & 061 & 062 & 063 & 066 & 065 & 067 \\
\hline Sup Sample Wt & 4.421 & & & \multirow{5}{*}{ not diluted } & & \\
\hline Sup $\mathrm{H}_{2} \mathrm{O}$ Added & 4.393 & & & & & \\
\hline Density Diluted & 1.210 & & & & & \\
\hline Density Undi] & $1.40^{6}$ & & & & & \\
\hline Diln Factor (v/v) & 2.4 & & & & & \\
\hline
\end{tabular}

${ }^{a}$ Cones fractured during heating, and some sample was lost. Weights before and after heating indicate extent of sample loss. See text (Section 3.1.3) for more details.

bstimated

Table 3-12. Analytical Results, Tank BY-106 Series Dissolution Test, $50^{\circ} \mathrm{C}$ (sample $\mathrm{A}$ is dilution-corrected; all results $\mu \mathrm{g} / \mathrm{mL}$ except as noted)

\begin{tabular}{|c|c|c|c|c|c|c|}
\hline Wt $\%$ Dilution: & $49 \%$ & $99 \%$ & $151 \%$ & $191 \%$ & $249 \%$ & $295 \%$ \\
\hline Supernate ID & BY6H-A & BY6H-B & BY6H-C & BY6H-D & BY6H-E & BY6H-F \\
\hline S98R000.. & 061 & 062 & 063 & 066 & 065 & 067 \\
\hline Density $(\mathrm{g} / \mathrm{mL})$ & 1.40 & 1.352 & 1.300 & 1.247 & 1.192 & 1.189 \\
\hline $\mathrm{TIC}$ & 6530 & 9370 & 6330 & 5120 & 4720 & 4030 \\
\hline $\mathrm{F}^{-}$ & 1370 & 2850 & 3730 & 2920 & 2490 & 1940 \\
\hline $\mathrm{Cl}$ & 2470 & 1500 & 1070 & 886 & 727 & 527 \\
\hline $\mathrm{NO}_{2}{ }^{-}$ & 33800 & 21800 & 16100 & 12700 & 10100 & 8360 \\
\hline $\mathrm{NO}_{3}{ }^{-3}$ & 420000 & 286000 & 226000 & 179000 & 139000 & 124000 \\
\hline $\mathrm{PO}_{4}{ }^{2-}$ & 3100 & 2330 & 1740 & 1410 & 1400 & 717 \\
\hline $\mathrm{SO}_{4}{ }^{--}$ & 2090 & 3050 & 6640 & 5780 & 4980 & 4010 \\
\hline $\mathrm{C}_{2} \mathrm{O}_{4}{ }^{2-}$ & $<1300$ & 1100 & 1490 & 1920 & 2700 & 3080 \\
\hline
\end{tabular}




\subsection{Tank B-106}

The aliquot of the composite sample taken for $\% \mathrm{H}_{2} \mathrm{O}$ analysis was labeled B6COMP, and was assigned Labcore number S98R000195. Analytical results were $58.0 \%$ by TGA and $44.2 \%$ by oven-drying. The difference between the two results is a bit disturbing. The oven-drying analysis was done two weeks after the TGA analysis, and the sample may have dried out during the intervening time if the sample vial was not tightly capped. The TGA result is in good agreement with the core sample analytical results as reported in the Tank Characterization Report (McCain 1996). The oven-drying result is not used in any of the mass balance calculations in this report.

\subsubsection{B-106 Sequential Dissolution Test}

Four $50 \mathrm{~mL}$ centrifuge cones were labeled B6-SEQA through B6-SEQD. The procedure was described in Section 2.2. Cones $A$ and $B$ were used for dilution with inhibited water $\left(0.01 \mathrm{M} \mathrm{NaOH}\right.$ and $\left.0.01 \mathrm{M} \mathrm{NaNO}_{2}\right)$. Cones $\mathrm{C}$ and $\mathrm{D}$ were used for dilution with $3 \mathrm{M} \mathrm{NaOH}$ solution. Weights, volumes, and analytical sample identification numbers of the various fractions are shown in Table 3-13. Sample analytical results are shown in Tables 3-14 and 3-15 for inhibited water diluent and $3 \mathrm{M}$ $\mathrm{NaOH}$ diluent, respectively.

It became clear from the results of the sequential dissolution test that the B-106 composite sample behaved more like a sludge than a saltcake. Relatively little of the sample dissolved in either water or $3 \mathrm{M} \mathrm{NaOH}$ at ambient temperature. Therefore, the series dilution tests performed for the other two tanks were not performed for tank B-106. 
Table 3-13. Raw Data, Tank B-106 Sequential Dissolution Test (weights in grams, volumes in $\mathrm{mL}$ )

\begin{tabular}{|c|c|c|c|c|}
\hline & B6-SEQA & B6-SEQB & B6-SEQC & B6-SEQD \\
\hline Sample Wt & 25.712 & 25.999 & 26.363 & 26.196 \\
\hline Diluent & inhibited $\mathrm{H}_{2} \mathrm{O}$ & inhibited $\mathrm{H}_{2} \mathrm{O}$ & $3 \mathrm{M} \mathrm{NaOH}$ & $3 \mathrm{M} \mathrm{NaOH}$ \\
\hline Wt Diluent Added & 25.012 & 24.981 & 27.070 & 26.983 \\
\hline Vol Diluent Added & 25.01 & 24.98 & 24.17 & 24.09 \\
\hline Total Vol & 43.8 & 44.5 & 43.8 & 44.0 \\
\hline Slurry Density & 1.16 & 1.15 & 1.22 & 1.21 \\
\hline Slurry Color & \multicolumn{2}{|c|}{ caramel } & \multicolumn{2}{|c|}{ red "BBQ sauce" } \\
\hline $1^{\text {st }}$ Wash Sample ID & \multicolumn{2}{|c|}{ B6QAB1 / S98R000225 } & \multicolumn{2}{|c|}{ B6QCD1 / S98R000226 } \\
\hline CSol Vol & 9.9 & 10.0 & 12.6 & 12.8 \\
\hline CSol Wt & 12.932 & 13.077 & 17.430 & 18.226 \\
\hline Wt Dil $\left(2^{\text {nd }}\right.$ Wash $)$ & 24.974 & 25.061 & 27.110 & 27.138 \\
\hline Total Vol & 35.0 & 35.0 & 37.0 & 37.5 \\
\hline $2^{\text {nd }}$ Wash Sample ID & \multicolumn{2}{|c|}{ B6QAB2 / S98R000228 } & \multicolumn{2}{|c|}{ B6QCD2 / S98R000229 } \\
\hline CSol Vol & 9.9 & 10.0 & 12.4 & 12.5 \\
\hline CSol Wt & 12.273 & 12.397 & 15.586 & 16.732 \\
\hline CSol Sample ID & \multicolumn{2}{|c|}{ B6QABS / S98R000231,3 } & \multicolumn{2}{|c|}{ B6QCDS / S98R000232, 4} \\
\hline
\end{tabular}


Table 3-14. Analytical Results, Tank B-106 Sequential Dissolution Test, Inhibited Water Diluent

\begin{tabular}{|c|c|c|c|}
\hline Analyte & $\begin{array}{c}\mathrm{I}^{\text {st }} \text { Water Wash } \\
\text { B6QAB 1 } \\
\text { S98R000225 } \\
(\mu \mathrm{g} / \mathrm{mL})\end{array}$ & $\begin{array}{c}2^{\text {nd }} \text { Water Wash } \\
\text { B6QAB2 } \\
\text { S98R000228 } \\
(\mu \mathrm{g} / \mathrm{mL})\end{array}$ & $\begin{array}{c}\text { CSol } \\
\text { B6QABS } \\
\text { S98R000231, } 3 \\
(\mu \mathrm{g} / \mathrm{g})\end{array}$ \\
\hline Al & $<5$ & 2 & 4740 \\
\hline $\mathrm{Bi}$ & $<10$ & $<4$ & 5160 \\
\hline $\mathrm{Ca}$ & $<10$ & $<4$ & 5060 \\
\hline $\mathrm{Cr}$ & 16 & 4 & 371 \\
\hline $\mathrm{Fe}$ & $<5$ & $<2$ & 27400 \\
\hline $\mathrm{K}$ & 103 & 25 & $<300$ \\
\hline $\mathrm{Mg}$ & $<10$ & $<4$ & 1190 \\
\hline $\mathrm{Mn}$ & $<1$ & $<1$ & 215 \\
\hline $\mathrm{Na}$ & 46400 & 13700 & 42500 \\
\hline$P$ & 3400 & 1180 & 28000 \\
\hline $\mathrm{Pb}$ & $<10$ & $<4$ & 667 \\
\hline $\mathrm{S}$ & 2880 & 787 & 745 \\
\hline $\mathrm{Si}$ & 7 & 3 & 355 \\
\hline $\mathrm{Sr}$ & $<1$ & $<1$ & 716 \\
\hline $\mathrm{U}$ & $<50$ & $<20$ & 19000 \\
\hline $\mathrm{F}$ & 524 & 534 & $\mathrm{na}^{\mathrm{a}}$ \\
\hline $\mathrm{Cl}^{-}$ & 701 & 174 & na \\
\hline $\mathrm{NO}_{2}^{-}$ & 5730 & 1600 & na \\
\hline $\mathrm{NO}_{3}{ }^{-}$ & 103000 & 26700 & na \\
\hline $\mathrm{PO}_{4}{ }^{3-}$ & 11300 & 3500 & na \\
\hline $\mathrm{SO}_{4}{ }^{2-}$ & 7240 & 2020 & na \\
\hline $\mathrm{C}_{2} \mathrm{O}_{4}{ }^{2-}$ & $<1070$ & $<117$ & na \\
\hline TIC & 162 & 64 & 112 \\
\hline TOC & 204 & 60 & 376 \\
\hline $\mathrm{OH}^{-}$ & $<2500$ & $<63$ & na \\
\hline${ }^{137} \mathrm{Cs}(\mu \mathrm{Ci} / \mathrm{mL})$ & 2.2 & 0.5 & na \\
\hline Density $(\mathrm{g} / \mathrm{mL})$ & 1.107 & 1.023 & na \\
\hline$\% \mathrm{H}_{2} \mathrm{O}$ & 84.1 & 95.7 & 76.7 \\
\hline
\end{tabular}

a na $=$ not analyzed 
Table 3-15. Analytical Results, Tank B-106 Sequential Dissolution Test, $3 \mathrm{M} \mathrm{NaOH}$ Diluent

\begin{tabular}{|c|c|c|c|}
\hline Analyte & $\begin{array}{c}1^{\text {st }} \text { Wash } \\
\text { B6QCD } \\
\text { S98R000226 } \\
(\mu \mathrm{g} / \mathrm{mL}) \\
\end{array}$ & $\begin{array}{c}2^{\text {ndd }} \text { Wash } \\
\text { B6QCD2 } \\
\text { S98R000229 } \\
(\mu \mathrm{g} / \mathrm{mL}) \\
\end{array}$ & $\begin{array}{c}\text { CSol } \\
\text { B6QCDS } \\
\text { S98R000232, } 4 \\
(\mu \mathrm{g} / \mathrm{g}) \\
\end{array}$ \\
\hline $\mathrm{Al}$ & 1340 & 519 & 741 \\
\hline $\mathrm{Bi}$ & $<20$ & $<20$ & 3490 \\
\hline$\overline{\mathrm{Ca}}$ & $<20$ & $<20$ & 3150 \\
\hline $\mathrm{Cr}$ & 30 & 16 & 216 \\
\hline $\mathrm{Fe}$ & $<10$ & $<10$ & 18500 \\
\hline $\mathrm{K}$ & 144 & $<100$ & $<300$ \\
\hline $\mathrm{Mg}$ & $<20$ & $<20$ & 790 \\
\hline $\mathrm{Mn}$ & $<2$ & $<2$ & 145 \\
\hline $\mathrm{Na}$ & 69600 & 70800 & 101000 \\
\hline $\mathrm{p}$ & 1660 & 1470 & 24400 \\
\hline $\mathrm{Pb}$ & $<20$ & $<20$ & 449 \\
\hline $\mathrm{S}$ & 3110 & 920 & 685 \\
\hline $\mathrm{Si}$ & 118 & 176 & 216 \\
\hline $\mathrm{Sr}$ & $<2$ & $<2$ & 475 \\
\hline $\mathrm{U}$ & $<100$ & $<100$ & 12400 \\
\hline $\mathrm{F}$ & 656 & 612 & $\mathrm{na}^{\mathrm{a}}$ \\
\hline $\mathrm{Cl}^{-}$ & 822 & 307 & na \\
\hline $\mathrm{NO}_{2}^{-}$ & 5710 & 1340 & na \\
\hline $\mathrm{NO}_{3}{ }^{-}$ & 111000 & 30500 & na \\
\hline $\mathrm{PO}_{4}{ }^{{ }^{-}}$ & 5880 & 4270 & na \\
\hline $\mathrm{SO}_{4}{ }^{2-}$ & 8480 & 2250 & na \\
\hline $\mathrm{C}_{2} \mathrm{O}_{4}{ }^{2-}$ & $<1070$ & $<117$ & na \\
\hline TIC & 205 & 159 & 301 \\
\hline TOC & 215 & 53 & 322 \\
\hline $\mathrm{OH}^{-}$ & 16700 & 39500 & na \\
\hline${ }^{137} \mathrm{Cs}(\mu \mathrm{Ci} / \mathrm{mL})$ & 10.3 & 3.3 & na \\
\hline Density $(\mathrm{g} / \mathrm{mL})$ & 1.140 & 1.127 & na \\
\hline$\% \mathrm{H}_{2} \mathrm{O}$ & 79.5 & 86.0 & 83.6 \\
\hline
\end{tabular}

${ }^{a}$ na $=$ not analyzed 


\subsection{DILUTION RESULTS, VOLUME AND WEIGHT MEASUREMENTS}

All results quoted in this section have been "normalized" to a common basis for comparison. The common basis is defined in each sub-section as appropriate.

\subsection{Tank BY-102}

The obvious, direct way to determine how much salt dissolved upon dilution is to measure the volume percent and/or weight percent centrifuged solids remaining after the dissolution. These values as measured during the series dilution tests are shown in Table 4-1. Entries for volume percent centrifuged solids (Vol\% CSol) and weight percent centrifuged solids ( $\mathrm{Wt} \% \mathrm{CSOl}$ ) are measured relative to the total volume and weight, respectively, of the diluted samples. Entries for "Wt $\%$ CSol relative to undiluted sample" are self-explanatory, and provide a more consistent way of visualizing the amount of sample remaining undissolved. The latter data are plotted in Figure 4-1, where the same values are expressed as "percent undissolved".

The undiluted composite sample was not centrifuged. Given the low weight percent water (approximately $26 \%$ - see Section 3.1 ) and the general appearance of the saltcake, centrifugation would be unlikely to produce any separation of a liquid phase. The Vol\% CSol and Wt\% CSol can be assumed to be $100 \%$.

Table 4-1. Percent Centrifuged Solids as a Function of Dilution, Tank BY-102

\begin{tabular}{|c|c|c|c|c|c|c|}
\hline \multicolumn{7}{|c|}{ at $25^{\circ} \mathrm{C}$} \\
\hline $\mathrm{Wt} \%$ Dilution $\rightarrow$ & 51 & 100 & 150 & 200 & 251 & 299 \\
\hline Vol $\%$ CSol & 67 & 32 & 16 & 9 & 6 & 5 \\
\hline $\mathrm{Wt} \% \mathrm{CSOl}$ & 72 & 38 & 17 & 11 & 8 & 7 \\
\hline $\begin{array}{l}\text { Wt\% CSol relative } \\
\text { to undiluted sample }\end{array}$ & 107 & 75 & 43 & 33 & 30 & 27 \\
\hline \multicolumn{7}{|c|}{ at $50^{\circ} \mathrm{C}$} \\
\hline $\mathrm{Wt} \%$ Dilution $\rightarrow$ & 50 & 99 & 148 & 198 & 249 & 297 \\
\hline Vol\% CSol & 38 & 22 & 10 & 5 & 4 & 2 \\
\hline $\mathrm{Wt} \% \mathrm{CSOl}$ & 48 & 25 & 13 & 10 & 7 & 3 \\
\hline $\begin{array}{l}\mathrm{Wt} \% \mathrm{CSol} \text { relative } \\
\text { to undiluted sample }\end{array}$ & 71 & 48 & 33 & $n a^{1}$ & $\mathrm{na}^{1}$ & 13 \\
\hline
\end{tabular}

${ }^{1}$ not available because unknown amount of sample was lost during heating. 


\section{Figure 4-1. Tank BY-102 Saltcake Dissolution}

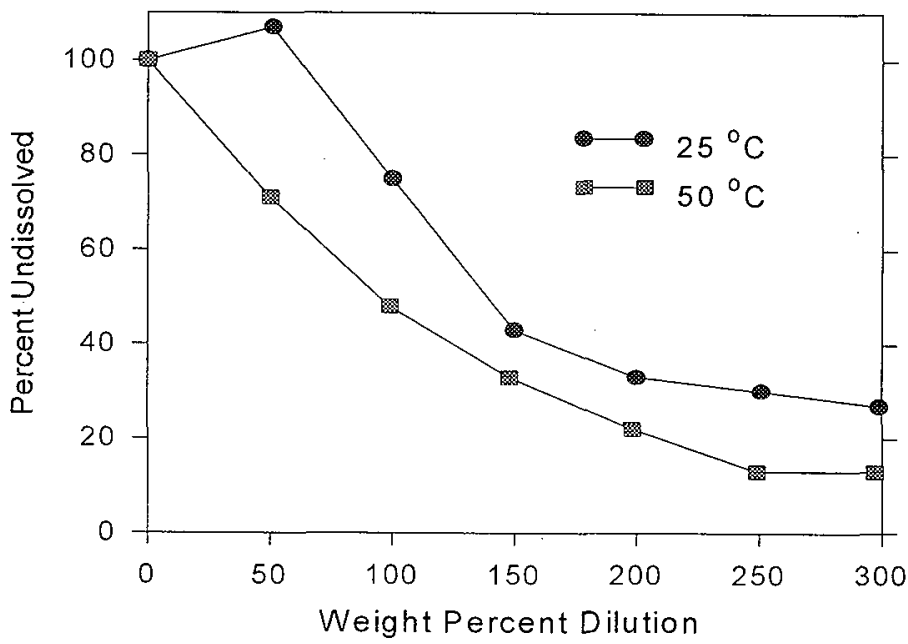

It is clear from the data that most of the saltcake sample is soluble in water. As much as $87 \%$ of the sample dissolved at $300 \%$ dilution by weight and $50{ }^{\circ} \mathrm{C}$. It is also clear that raising the temperature from $25^{\circ} \mathrm{C}$ to $50^{\circ} \mathrm{C}$ had a dramatic impact on the overall solubility of the saltcake. The fact that the weight percent centrifuged solids was $107 \%$ relative to the undiluted sample at $50 \%$ dilution, $25{ }^{\circ} \mathrm{C}$, is not surprising. The centrifuged solids from the diluted sample retained more interstitial liquid than the initial composite sample had.

\subsection{Tank BY-106}

The percent centrifuged solids values for the tank BY-106 series dilution tests are shown in Table 4-2. Again, the undiluted composite sample was never centrifuged. Given the very low weight percent water (14.7\% - see Section 3.2) and the general appearance of the saltcake, centrifugation would be unlikely to produce any separation of a liquid phase. The Vol\% CSol and Wt $\%$ CSol can be assumed to be $100 \%$. The "percent undissolved" values, or Wt\% CSol relative to undiluted sample, are plotted in Figure 4-2. 
HNF-3437, Rev. 0

Table 4-2. Percent Centrifuged Solids as a Function of Dilution, Tank BY-106

\begin{tabular}{|c|c|c|c|c|c|c|}
\hline \multicolumn{7}{|c|}{ at $25^{\circ} \mathrm{C}$} \\
\hline $\mathrm{Wt} \%$ Dilution $\rightarrow$ & 46 & 99 & 164 & 204 & 246 & 292 \\
\hline Vol\% CSol & 41 & 11 & 5 & 4 & 2 & 1 \\
\hline $\mathrm{Wt} \%$ CSol & 47 & 13 & 7.7 & 6.4 & 4.5 & 4.1 \\
\hline $\begin{array}{l}\mathrm{Wt} \% \text { CSol relative } \\
\text { to undiluted sample }\end{array}$ & 70 & 27 & 21 & 19 & 16 & 16 \\
\hline \multicolumn{7}{|c|}{ at $50^{\circ} \mathrm{C}$} \\
\hline Wt\% Dilution $\rightarrow$ & 49 & 99 & 151 & 191 & 249 & 295 \\
\hline Vol\% CSol & 27 & 12 & 3 & 3 & 2 & 1 \\
\hline $\mathrm{Wt} \% \mathrm{CSOl}$ & 34 & 13 & 5 & 5 & 2.2 & 1.4 \\
\hline $\begin{array}{l}\text { Wt\% CSol relative } \\
\text { to undiluted sample }\end{array}$ & 50 & 19 & 11 & 10 & 6.5 & 4.5 \\
\hline
\end{tabular}

Even more of the saltcake sample is soluble in water than for the BY-102 saltcake. Over $95 \%$ of the sample dissolved at $300 \%$ dilution by weight and $50{ }^{\circ} \mathrm{C}$. That indicates that, ideally, over $95 \%$ of the saltcake could be retrieved from the tank as supernatant liquid under similar dissolution conditions. As with BY-102, raising the temperature from $25^{\circ} \mathrm{C}$ to $50^{\circ} \mathrm{C}$ had a dramatic impact on the overall solubility of the saltcake. 
HNF-3437, Rev. 0

Figure 4-2. Tank BY-106 Saltcake Dissolution

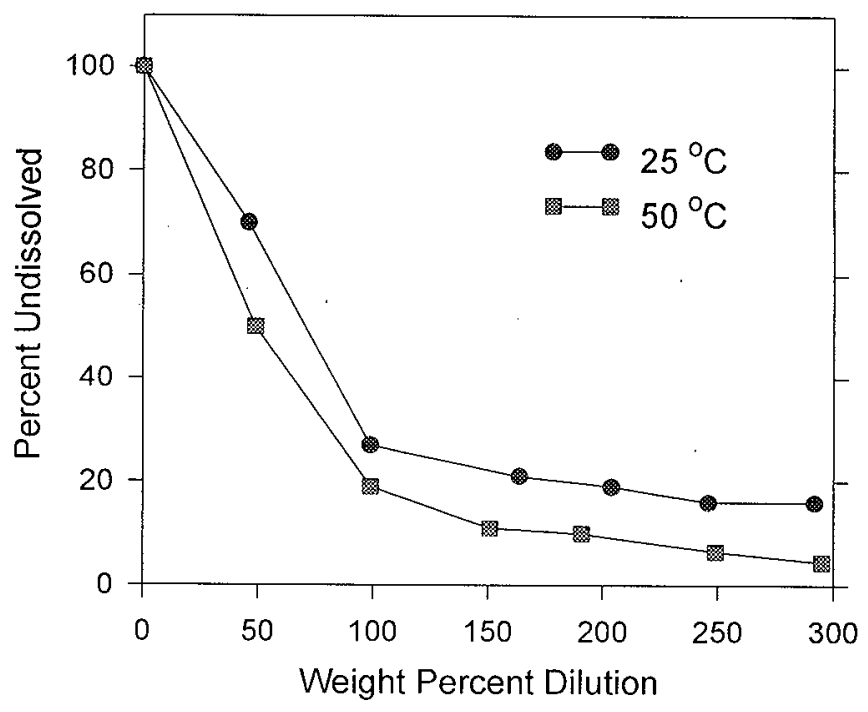




\subsection{CHEMICAL COMPOSITION TRENDS}

The gross sample behavior explored in the previous section (i.e., volume and weight of centrifuged solids as a function of dilution) can be understood in terms of what happens to individual saltcake components. Most of the trends described in the following sections can be observed in the "Analytical Results" tables in Section 3.

Certain components can be assumed, based on prior solubility studies on Hanford waste samples (Person 1996, Herting 1997, 1998), to be completely soluble under all dilution conditions. These components include chloride, potassium, and - for the current cases - nitrite. Solubilities of other components can often be evaluated by comparing concentration trends with these completely soluble components.

\subsection{Tank BY-102}

Liquid phase concentration data for chloride, fluoride and oxalate are shown in Figure 5-1.

The chloride data are typical of the trends expected for a component that is fully soluble at all dilution levels. The concentration in solution falls with increasing dilution in a predictable pattern (see discussion of nitrite below). Presumably, all of the chloride is in solution at $50 \%$ dilution for both temperatures. The total liquid volume, though, is smaller at $25^{\circ} \mathrm{C}$ because there are fewer total salts dissolved, as evidenced by the volume of centrifuged solids $\left(67 \%\right.$ at $25^{\circ} \mathrm{C}$ vs. $38 \%$ at $50^{\circ} \mathrm{C}$, cf. Table $\left.4-1\right)$. Therefore, the chloride concentration is significantly higher at $25^{\circ} \mathrm{C}$ than at $50^{\circ} \mathrm{C}$. The concentration curves for the two temperatures converge at higher dilution because the liquid volumes are less different after the major salts have dissolved.

The fluoride data, in contrast, show the behavior of a sparingly soluble component that becomes increasingly soluble as the ionic strength of the solution drops with dilution. Fluoride concentration rises with dilution from 50 to $200 \%$ dilution, then begins to fall with further dilution. The shape of the curve in Figure 1 implies that there are fluoride-containing solids present until approximately $200-250 \%$ dilution. The solid phase responsible for controlling the behavior of the fluoride ion is open to speculation at this point. Candidates include $\mathrm{NaF}, \mathrm{Na} 3 \mathrm{FSO} 4$, and $\mathrm{Na}_{7} \mathrm{~F}\left(\mathrm{PO}_{4}\right)_{2} \cdot 19 \mathrm{H}_{2} \mathrm{O}$, and indeed a mixture of the candidates is possible. The solubility of the fluoride-phosphate double salt is known to be very temperature dependent (Beahm 1998), and may be responsible for the large difference between the two temperature points at $50 \%$ dilution. To further the same argument, at $100-200 \%$ dilution all of the fluoride-phosphate double salt would be dissolved, and the solubility of the remaining solid phase(s) would have little or no dependence on temperature. Solubility dependence on ionic strength remains high, though, implicating the fluoride-sulfate double salt. The presence of this salt is further implied by the sulfate data (vide infra). 
HNF-3437, Rev. 0

\section{Figure 5-1. Tank BY-102 Chloride, Fluoride, Oxalate}

(open symbols $25^{\circ} \mathrm{C}$; closed symbols $50^{\circ} \mathrm{C}$ )

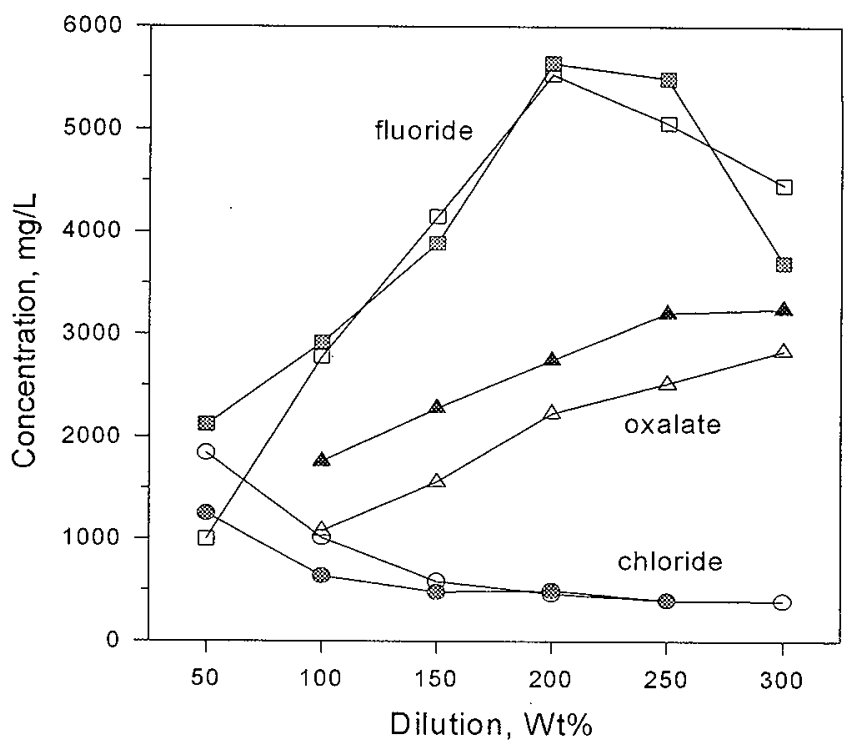

The oxalate data show a continual increase in liquid phase concentration with dilution throughout the dilution range. The undissolved solids at $250 \%$ dilution still include sodium oxalate, and probably at $300 \%$ dilution as well. The effect of temperature on solubility of sodium oxalate has been studied with simulated waste solutions over the temperature range of 25 to $50^{\circ} \mathrm{C}$ (Barney 1997). The observed solubility was lowest at $25^{\circ} \mathrm{C}$ and highest at $30^{\circ} \mathrm{C}$. Under conditions of $\mathrm{pH}$ and ionic strength roughly matching the current conditions, the Barney data show sodium oxalate solubility approximately $20 \%$ higher at $50{ }^{\circ} \mathrm{C}$ than at $25^{\circ} \mathrm{C}$. On an absolute scale, however, the agreement between the current data and the Barney data is not good - the current data are approximately one order of magnitude higher in oxalate concentration than the Barney data. 
Figure 5-2 shows dilution trends for nitrite and carbonate (measured as TIC). The nitrite data are typical of a component (sodium nitrite) that is fully soluble at all dilution levels. At $50 \%$ dilution, nitrate concentration is higher at $25^{\circ} \mathrm{C}$ than at $50^{\circ} \mathrm{C}$ because of the smaller solution volume at $25^{\circ} \mathrm{C}$, as discussed above for chloride. The temperature curves converge at higher dilution. The $50^{\circ} \mathrm{C}$ data are plotted in Figure $5-3$ as the ratio of the concentration at $50 \%$ dilution $\left(\mathrm{C}_{0}\right)$ to the concentration at each higher dilution level (C). The line in Figure 5-3 is a first-order regression fit to the data. Points should follow this linear pattern if the only effect from one dilution level to another is, in fact, simple dilution of the liquid. Any dissolution of solids occurring with dilution would cause the points to deviate from the linear relationship. Nitrate data are not plotted. They follow the same pattern as the nitrite data, but at a higher concentration level.

Figure 5-2. Tank BY-102 Nitrite, TIC (open symbols $25^{\circ} \mathrm{C}$; closed symbols $50^{\circ} \mathrm{C}$ )

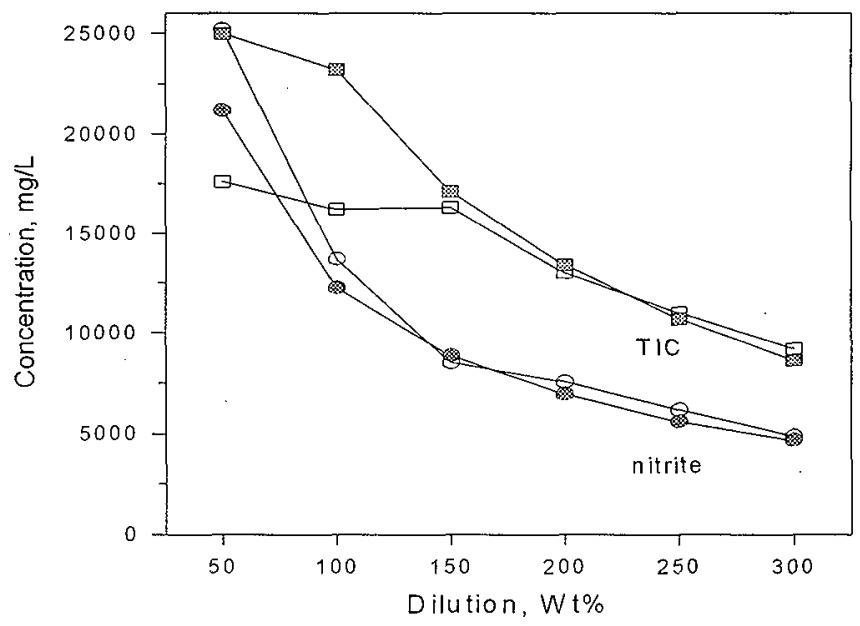

The carbonate (TIC) data follow the same simple dilution behavior above $150 \%$ dilution. At lower dilutions, there is evidence of the presence of solid-phase sodium carbonate. The fact that the solubility is significantly higher at $50{ }^{\circ} \mathrm{C}$ than at $25^{\circ} \mathrm{C}$ implies that the carbonate salt is hydrated, since the solubility of the anhydrous form of sodium carbonate exhibits inverse temperature dependence. 
Figure 5-3. TankBY-102 Nitrite Ratio Plot, $50^{\circ} \mathrm{C}$

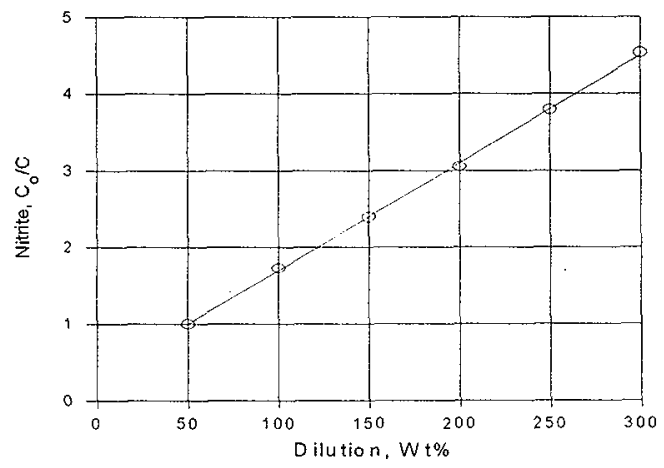

Figure 5-4 shows the dilution trends for phosphate and sulfate. The phosphate solubility is more temperature-dependent than any of the other salts. It is surprising, though, that the solubility doesn't increase more than it does with increasing dilution. The large temperature effect points to the double salt $\mathrm{Na}_{7} \mathrm{~F}\left(\mathrm{PO}_{4}\right)_{2} \cdot 19 \mathrm{H}_{2} \mathrm{O}$ as the solid phase, but the lack of dilution effect suggests that this is not the right species.

Figure 5-4. Tank B Y-102 Phosphate, Sulfate

(open symbols $25^{\circ} \mathrm{C}$; closed symbols $50^{\circ} \mathrm{C}$ )

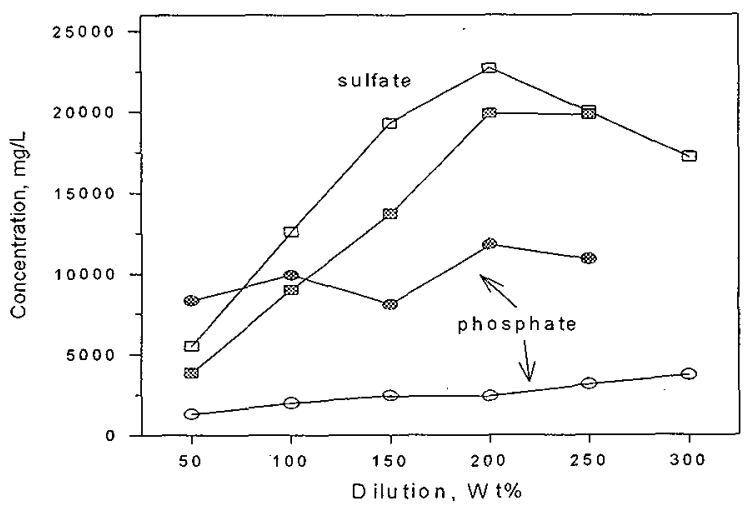


The sulfate data parallel the fluoride data quite well. Concentration peaks at $200 \%$ dilution in both cases, and the sulfate:fluoride ratio is approximately 4 , compared to 5.05 for the ideal ratio if the salt $\mathrm{Na} 3 \mathrm{FSO} 4$ contributed all of the sulfate and fluoride. The assumption that the fluoride-phosphate double salt would also add to the fluoride level is consistent with the observed ratio being slightly lower than the ideal.

The assumption that all fluoride comes from fluoride-phosphate and fluoridesulfate double salts provides a remarkably good fit to the observed fluoride data. At $25{ }^{\circ} \mathrm{C}$ and $50 \%$ dilution, the $1310 \mathrm{mg} / \mathrm{L}$ phosphate would account for $130 \mathrm{mg} / \mathrm{L}$ fluoride; $5520 \mathrm{mg} / \mathrm{L}$ sulfate would add $1090 \mathrm{mg} / \mathrm{L}$ fluoride; total fluoride predicted would be 1220 $\mathrm{mg} / \mathrm{L}$; observed fluoride was $1000 \mathrm{mg} / \mathrm{L}$. Similar calculations at the other dilution/temperature levels result in the data presented in Table 5-1. The agreement between predicted and observed fluoride concentrations is compelling evidence for the presence of both fluoride double salts. The fact that the observed values are consistently slightly higher than the predicted values is evidence of a third salt, probably $\mathrm{NaF}$.

Table 5-1. Fluoride Concentrations Predicted from Phosphate and Sulfate Data, Tank BY-102

\begin{tabular}{|c|c|c|c|c|}
\hline \multirow{2}{*}{$\begin{array}{c}\text { Dilution, } \\
\text { Wt\% }\end{array}$} & \multicolumn{2}{|c|}{$25^{\circ} \mathrm{C}$} & \multicolumn{2}{c|}{$50^{\circ} \mathrm{C}$} \\
\cline { 2 - 5 } & Predicted & Observed & Predicted & Observed \\
\hline 50 & 1220 & 999 & 1590 & 2120 \\
\hline 100 & 2690 & 2780 & 2780 & 2920 \\
\hline 150 & 4060 & 4150 & 3530 & 3890 \\
\hline 200 & 4740 & 5530 & 5130 & 5640 \\
\hline 250 & 4270 & 5060 & 5010 & 5490 \\
\hline 300 & 3780 & 4460 & -- & -- \\
\hline
\end{tabular}


HNF-3437, Rev. 0

\subsection{Tank BY-106}

Saltcake from tank BY-106 contains approximately ten times as much sodium nitrate as any other salt. The $\mathrm{NaNO}_{3}$ dissolves readily in water. Figure $5-5$ shows the concentration of nitrate in the liquid phase as a function of dilution. At $25{ }^{\circ} \mathrm{C}$, there are clearly undissolved $\mathrm{NaNO}_{3}$ solids at $50 \%$ dilution, but no solids remain beyond $100 \%$ dilution. At $50{ }^{\circ} \mathrm{C}$, there is probably a trace of solid $\mathrm{NaNO}_{3}$ remaining at $50 \%$ dilution. The data from $100 \%$ to $300 \%$ dilution can be plotted as the concentration ratio $\mathrm{C}_{2} / \mathrm{C}$, similar to Figure 5-3. (The nitrate plot is not shown.) In this case, the concentration at $100 \%$ dilution is defined as $\mathrm{C}_{0}$. The resulting regression line can be used to predict the concentration at $50 \%$ dilution. The predicted concentration, based on the $50^{\circ} \mathrm{C}$ data, is $477,000 \mathrm{mg} / \mathrm{L}$, which is only a little higher than the $420,000 \mathrm{mg} / \mathrm{L}$ observed (Table 3-12).

Figure 5-5. Tank BY-106 Nitrate Concentrations

(open symbols $25^{\circ} \mathrm{C}$; closed symbols $50^{\circ} \mathrm{C}$ )

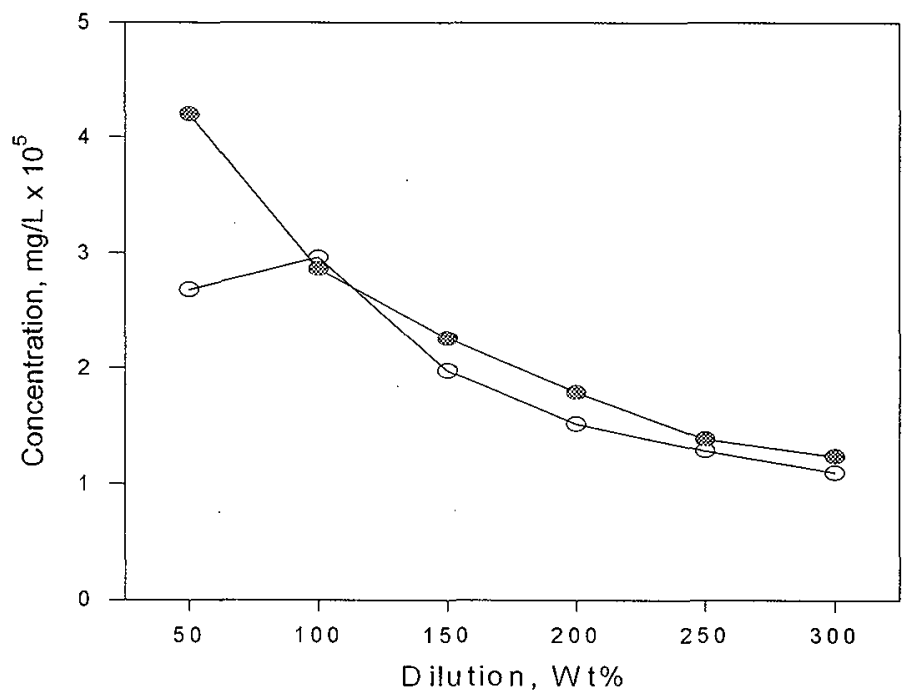


Other components follow dilution patterns similar or analogous to those in waste from tank BY-102. Figure 5-6 shows the nitrite and TIC data. Nitrite is unsaturated at all dilution levels. Carbonate appears to be unsaturated at all levels at $25^{\circ} \mathrm{C}$, but there are undissolved solids at $50 \%$ dilution and $50^{\circ} \mathrm{C}$. If the anhydrous sodium carbonate exists under the latter conditions, then it is likely that its solubility would be lower than the solubility of the hydrated salt was at $25^{\circ} \mathrm{C}$, so the observed data are not inconsistent. However, the possibility of an analytical error in either the 25 or $50^{\circ} \mathrm{C}$ data at $50 \%$ dilution can't be ruled out.

Figure 5-6: Tank BY-106 Nitrite, TIC

(open symbols $25^{\circ} \mathrm{C}$; filled symbols $50^{\circ} \mathrm{C}$ )

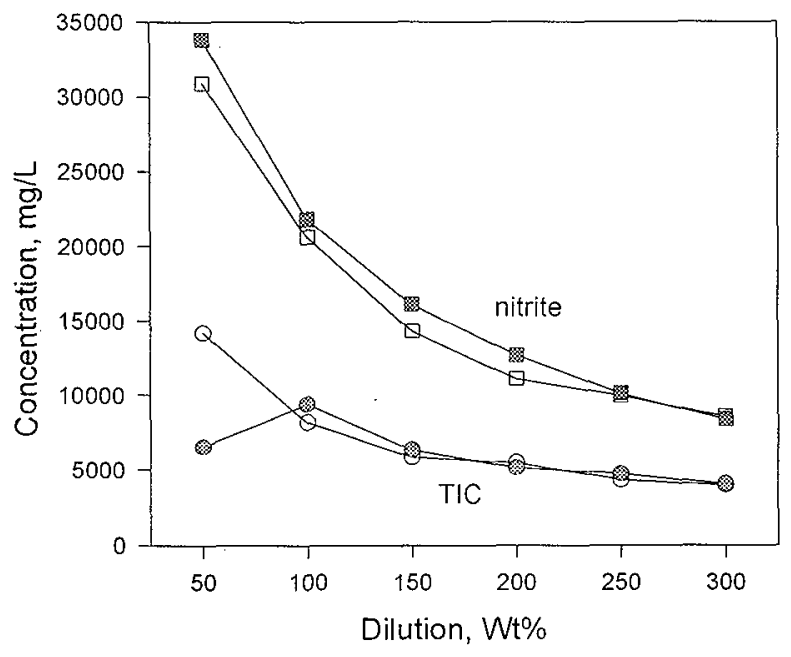

Figure 5-7 shows the concentration data for fluoride, phosphate and sulfate. Trends for fluoride and sulfate are parallel, but the fluoride concentration is two to four times higher than what would be predicted from the contributions from fluoride-sulfate and fluoride-phosphate double salts. Apparently, much or most of the fluoride in BY-106 waste comes from the simple $\mathrm{NaF}$ salt. 


\section{Figure 5-7. Tank BY-106 Fluoride, Phosphate, Sulfate}

(open symbols $25^{\circ} \mathrm{C}$; filled symbols $50^{\circ} \mathrm{C}$ )

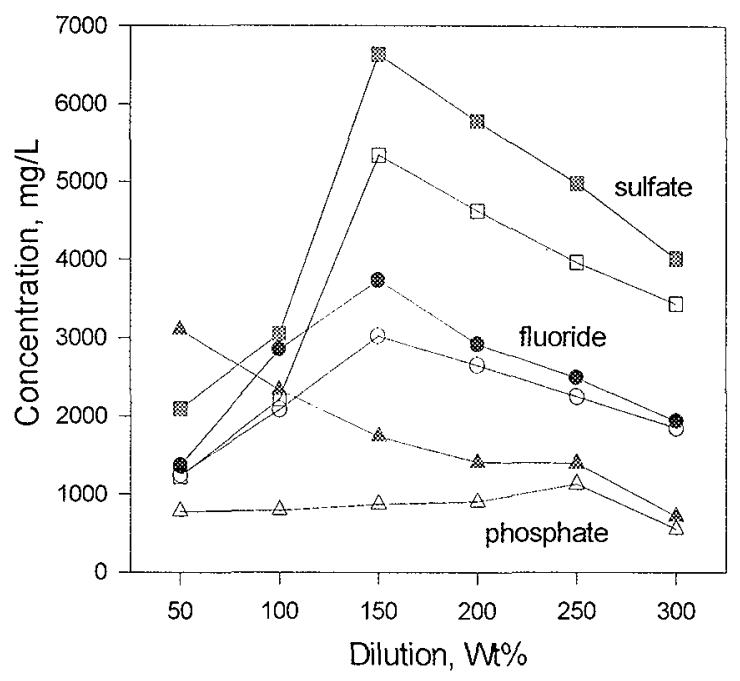

The temperature dependence of the sulfate concentration is rather perplexing, especially when comparing the results for BY-102 and BY-106. In BY-102 waste (see Figure 5-4), the sulfate was more soluble at $25^{\circ} \mathrm{C}$, and in $\mathrm{BY}-106$ waste the sulfate is more soluble at $50^{\circ} \mathrm{C}$. Furthermore, for BY-106, the two curves for the different temperatures should converge at high dilution where [presumably] all of the sulfate is dissolved, but they do not. 
Sodium oxalate solubility (Figure 5-8) increases steadily with dilution and falling ionic strength, as expected. The concentrations are approximately the same at all dilutions as those found for the tank BY-102 study. The chloride data (also Figure 5-8) show the same perplexing temperature differences noted above for sulfate. With all of the chloride in solution at all dilution levels, the temperature curves should nearly coincide, except for differences in solution volumes which should be relatively small. There is no apparent reason for the chloride concentration to be higher at $50^{\circ} \mathrm{C}$ than at $25{ }^{\circ} \mathrm{C}$.

\section{Figure 5-8. Tank BY-106 Chloride, Oxalate}

(open symbols $25^{\circ} \mathrm{C}$; filled symbols $50^{\circ} \mathrm{C}$ )

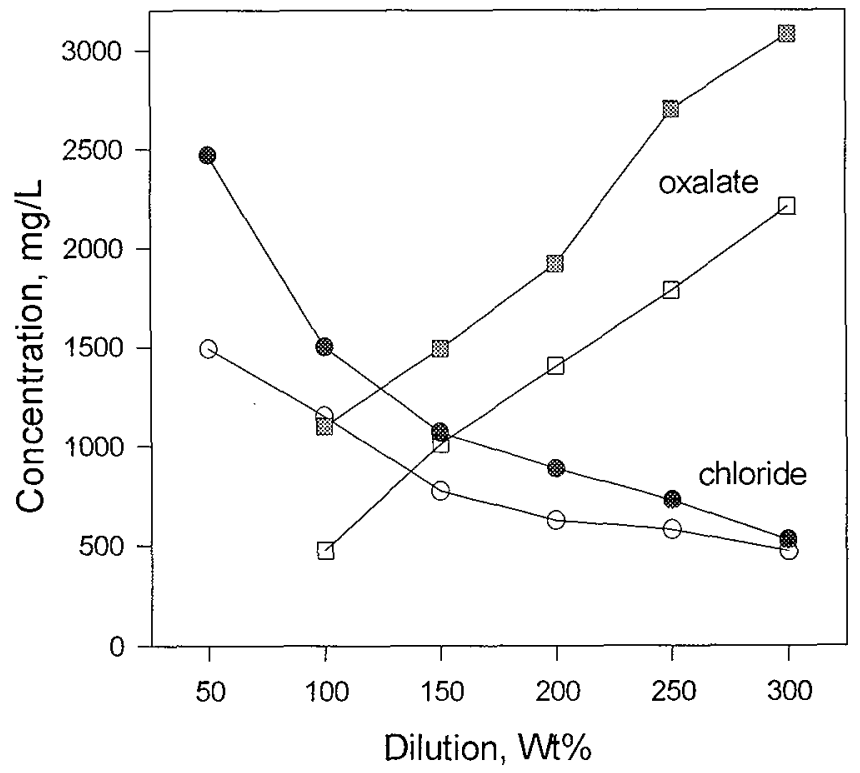


HNF-3437, Rev. 0

\subsection{POLARIZED LIGHT MICROSCOPY}

The polarized light microscope (PLM) provides a convenient tool for identifying solid crystalline phases in waste samples. Crystal size and shape (morphology) and optical properties (number of unique refractive indexes, approximate birefringence, extinction position) can be determined by simple microscopic observation of the crystals with polarized light. Each crystalline phase has a "fingerprint" set of physical and optical properties that make it identifiable virtually at a glance. Several types of crystalline phases common to Hanford waste have been fingerprinted this way and catalogued (Herting 1992).

\subsection{Tank BY-102}

As noted previously, all of the $50^{\circ} \mathrm{C}$ liquid samples from the tank BY-102 series dilution test formed crystals when the liquids cooled to ambient temperature. One drop of slurry from each of these samples was examined by PLM. In all cases, the crystals were isotropic (only one refractive index) and octahedral (belonging to the cubic crystal system). The size varied from sample to sample (see Figures 6-1 and 6-2). Crystals matching these properties have previously been identified as the fluoride-phosphate double salt $\mathrm{Na}_{7} \mathrm{~F}\left(\mathrm{PO}_{4}\right)_{2} \cdot 19 \mathrm{H}_{2} \mathrm{O}$. Sodium fluoride $(\mathrm{NaF})$ crystals have similar properties, but have not been observed to precipitate in such quantity from cooled solutions, as the double salt crystals have been (Herting 1996).

\subsection{Tank BY-106}

The liquid from the second wash step in the sequential dissolution test on BY-106 waste was expected to be saturated in sodium oxalate, but not in any other sodium salts. A portion of the wash liquid was evaporated in an open beaker on a magnetic stirplate until crystals began to form. A drop of the slurry was examined by PLM. The crystals (Figure 6-3) were elongated rods with moderate birefringence. They were positively identified as sodium oxalate by precipitating known sodium oxalate crystals under similar conditions in a simulated waste matrix. Further evaporation of the wash liquid produced a mixture of several types of crystals including $\mathrm{NaNO}_{3}, \mathrm{Na}_{2} \mathrm{CO}_{3} \cdot \mathrm{H}_{2} \mathrm{O}$, and some unidentified crystals. Further development work on identification of crystalline phases by a combination of PLM and x-ray analysis is planned for next fiscal year. 
HNF-3437, Rev. 0

Figure 6-1. Crystals Formed when BY-102 Liquid Cooled from $50^{\circ} \mathrm{C}$ to Ambient Temperature; Examples of Smaller Crystals

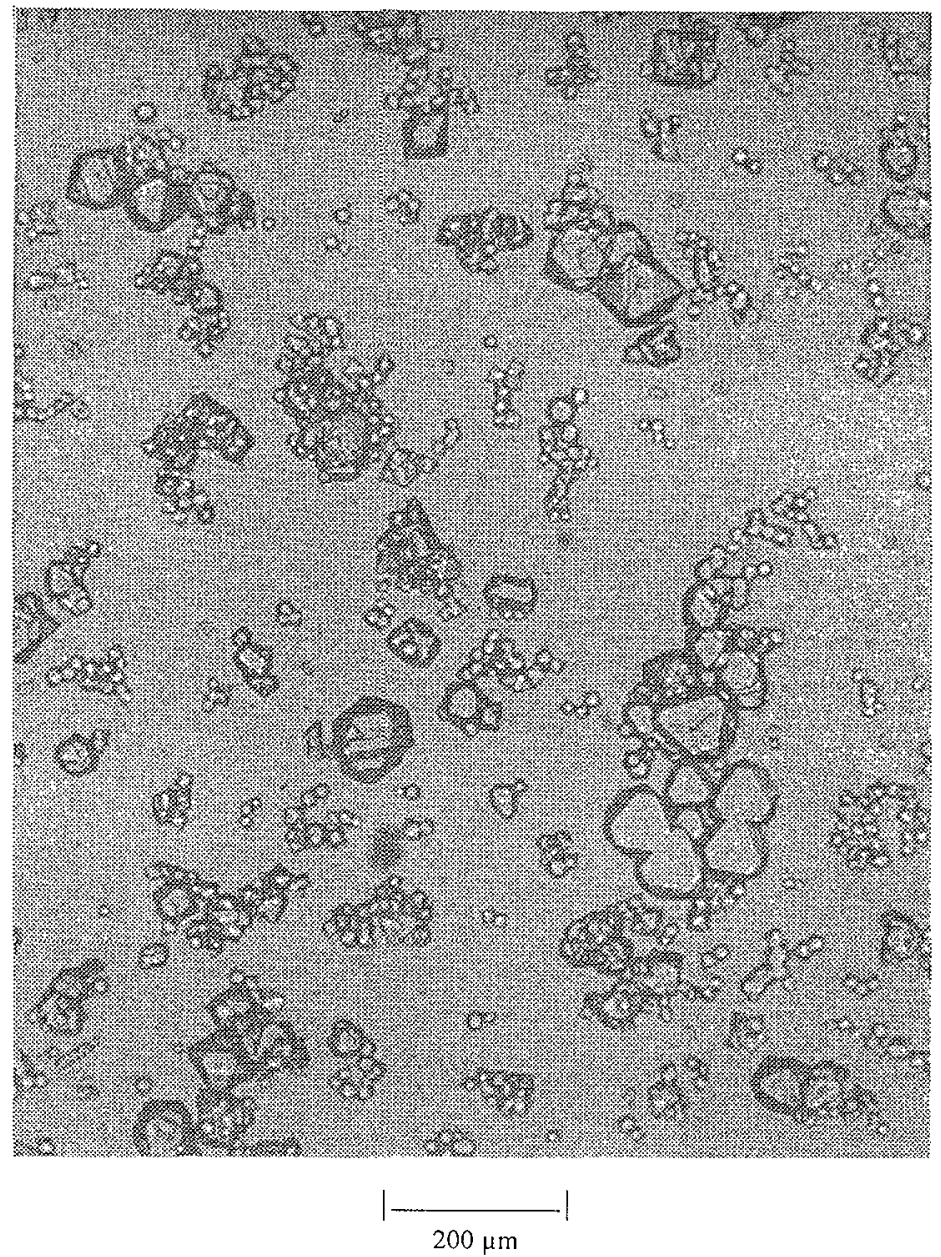

crystals identified as sodium fluoride diphosphate, $\mathrm{Na}_{7} \mathrm{~F}\left(\mathrm{PO}_{4}\right)_{2} \cdot 19 \mathrm{H}_{2} \mathrm{O}$ 
Figure 6-2. Crystals Formed when BY-102 Liquid Cooled from $50{ }^{\circ} \mathrm{C}$ to Ambient Temperature; Examples of Larger Crystals

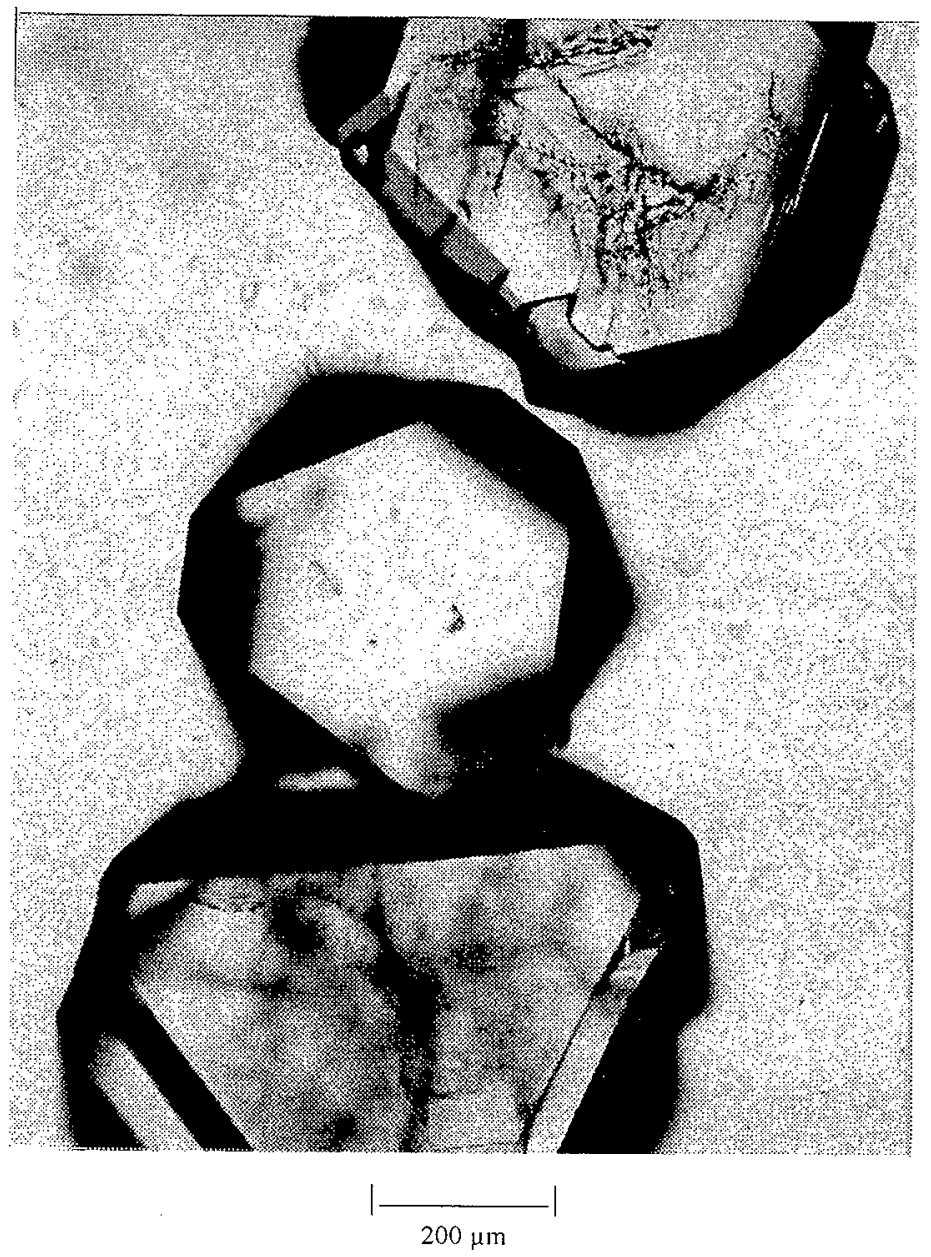

crystals identified as sodium fluoride diphosphate, $\mathrm{Na}_{7} \mathrm{~F}\left(\mathrm{PO}_{4}\right)_{2} \cdot 19 \mathrm{H}_{2} \mathrm{O}$ 
HNF-3437, Rev. 0

Figure 6-3. Crystals Formed by Evaporation of BY-106 Liquid from 2nd Water Wash

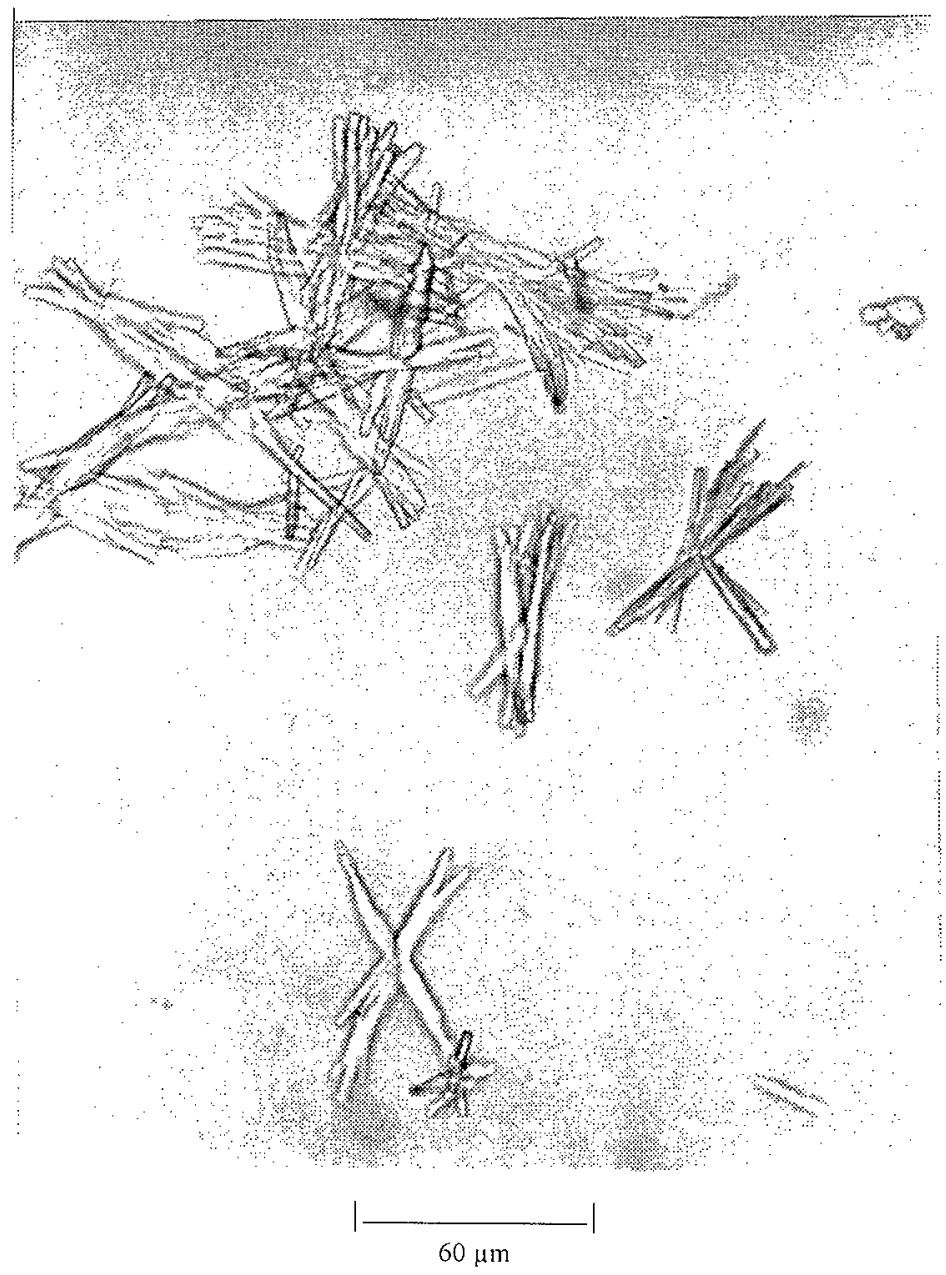

crystals identified as sodium oxalate, $\mathrm{Na}_{2} \mathrm{C}_{2} \mathrm{O}_{4}$ 


\subsection{PHASE DISTRIBUTION ANALYSIS}

The analytical data from the sequential dissolution tests can be used to calculate bulk waste compositions and solid/liquid phase distributions at $100 \%$ dilution and ambient temperature.

\subsection{Tank BY-102}

\subsubsection{Physical Sample Data}

Table 7-1 contains a summary of the physical measurement data used to calculate the bulk composition and phase distributions. Columns labeled "SEQA" and "SEQB" contain data recorded for each individual [duplicate] centrifuge cone. The column labeled "Total" represents either the sum of the individual cone entries or the analysis of the sample created by combining the contents of the two cones (see Section 2.2).

Table 7-1. Summary of Measurements from BY-102 Sequential Dissolution Test (weights in grams, volumes in $\mathrm{mL}$ )

\begin{tabular}{|c|c|c|c|}
\hline Measurement & SEQA & SEQB & Total \\
\hline Sample Weight & 30.670 & 32.183 & 62.853 \\
\hline $\mathrm{Wt} \mathrm{H}_{2} \mathrm{O}$ Added & 29.310 & 30.278 & 59.588 \\
\hline $\mathrm{Wt} 1^{\text {st }} \mathrm{H}_{2} \mathrm{O}$ Wash & 36.985 & 46.239 & 83.224 \\
\hline Vol $1^{\text {st }} \mathrm{H}_{2} \mathrm{O}$ Wash & 29.0 & 36.0 & 65.0 \\
\hline Density $1^{\text {st }} \mathrm{H}_{2} \mathrm{O}$ Wash & & & 1.232 \\
\hline$\% \mathrm{H}_{2} \mathrm{O}^{\mathrm{st}} \mathrm{H}_{2} \mathrm{O}$ Wash & & & $69.0^{\mathrm{a}}$ \\
\hline Wt Centrifuged Solids & 22.995 & 16.222 & 39.127 \\
\hline Wt $\mathrm{H}_{2} \mathrm{O}$ Added & 30.171 & 29.160 & 59.331 \\
\hline Wt $2^{\text {nd }} \mathrm{H}_{2} \mathrm{O}$ Wash & 44.538 & 36.514 & 81.052 \\
\hline Vol $2^{\text {nd }} \mathrm{H}_{2} \mathrm{O}$ Wash & 38.5 & 32.0 & 70.5 \\
\hline Density $2^{\text {nd }} \mathrm{H}_{2} \mathrm{O}$ Wash & & & 1.125 \\
\hline$\% \mathrm{H}_{2} \mathrm{O}_{2}{ }^{\text {nd }} \mathrm{H}_{2} \mathrm{O}$ Wash & & & 84.0 \\
\hline Final Wt Centrifuged Solids & 8.628 & 8.868 & 17.496 \\
\hline$\% \mathrm{H}_{2} \mathrm{O}$ Centrifuged Solids & & & 56.97 \\
\hline
\end{tabular}

${ }^{\text {a }}$ corresponds to $48 \% \mathrm{H}_{2} \mathrm{O}$ in centrifuged solids after $1^{\text {st }}$ wash (which wasn't measured)

\subsubsection{Composition by Fractions}

Table 7-2 shows the weight in grams of each component in the $1^{\text {st }}$ water wash, $2^{\text {nd }}$ water wash, centrifuged solids, and total sample (sum of the three fractions). The column 
labeled "Wt\%" is the total sample value for each component divided by the total sample weight from Table 7-1. The weights in each wash solution are found by multiplying the analytical result (Table 3-2) by the wash solution volume (Table 7-1). Similarly, the CSol weight come from the analytical result (again Table 3-2) times the final weight of centrifuged solids (Table 7-1).

Table 7-2. Tank BY-102 Analysis by Fractions ${ }^{1}$ (all values in grams)

\begin{tabular}{|c|c|c|c|c|c|}
\hline Analyte & $1^{\text {st }}$ Wash & $2^{\text {nd }}$ Wash & CSol & Total & $W t \%$ \\
\hline $\mathrm{A} 1$ & 0.57 & 0.09 & 0.38 & 1.04 & 1.65 \\
\hline $\mathrm{Ca}$ & & & 0.027 & 0.027 & 0.04 \\
\hline $\mathrm{Cr}$ & 0.072 & 0.012 & 0.041 & 0.125 & 0.20 \\
\hline $\mathrm{Fe}$ & & & 0.033 & 0.033 & 0.05 \\
\hline $\mathrm{K}$ & 0.086 & & & 0.086 & 0.14 \\
\hline $\mathrm{Mn}$ & & & 0.015 & 0.015 & 0.02 \\
\hline $\mathrm{Na}$ & 10.14 & 4.94 & 2.38 & 17.45 & 27.8 \\
\hline $\mathrm{Ni}$ & & & 0.017 & 0.017 & 0.03 \\
\hline $\mathrm{P}$ & 0.039 & 0.081 & 0.51 & 0.63 & 1.00 \\
\hline$S$ & 0.23 & 0.95 & 0.094 & 1.28 & 2.04 \\
\hline $\mathrm{Si}$ & 0.017 & 0.010 & 0.019 & 0.045 & 0.07 \\
\hline $\mathrm{U}$ & & & 0.040 & 0.040 & 0.06 \\
\hline $\mathrm{F}$ & 0.18 & 0.56 & na & 0.74 & 1.17 \\
\hline $\mathrm{Cl}$ & 0.069 & & na & 0.069 & 0.11 \\
\hline $\mathrm{NO}_{2}{ }^{-}$ & 0.96 & 0.20 & na & 1.16 & 1.85 \\
\hline $\mathrm{NO}_{3}{ }^{-}$ & 6.00 & 0.87 & na & 6.87 & 10.9 \\
\hline $\mathrm{PO}_{4}{ }^{2-}$ & 0.11 & 0.27 & na & 0.38 & 0.60 \\
\hline $\mathrm{SO}_{4}{ }^{-2}$ & 0.68 & 2.54 & na & 3.22 & 5.12 \\
\hline $\mathrm{C}_{2} \mathrm{O}_{4}{ }^{{ }^{-}}$ & 0.06 & 0.27 & $0.20^{4}$ & 1.20 & 1.99 \\
\hline $\mathrm{TIC}$ & 2.13 & 0.42 & 0.06 & 2.60 & 4.13 \\
\hline $\mathrm{TOC}$ & 0.066 & 0.078 & 0.25 & 0.40 & 0.63 \\
\hline $\mathrm{OH}^{-}$ & 0.59 & 0.10 & na & 0.69 & 1.09 \\
\hline${ }^{137} \mathrm{Cs}(\mathrm{mCi})$ & 2.35 & 0.44 & na & 2.79 & -- \\
\hline $\mathrm{H}_{2} \mathrm{O}$ & 57.42 & 68.08 & 9.97 & 16.68 & 26.5 \\
\hline $\begin{array}{l}\text { Total Mass } \\
\text { Calculated }^{2}\end{array}$ & 88.4 & 80.1 & 16.3 & 65.8 & 104.6 \\
\hline $\begin{array}{l}\text { Total Mass } \\
\text { Measured }^{3}\end{array}$ & 83.2 & 81.1 & 17.5 & 62.9 & 100.0 \\
\hline
\end{tabular}

${ }^{1}$ Blank entries indicate analytical result was below detection limit.

${ }^{2}$ Sum of individual components (see text for details).

${ }^{3}$ Actual weights of wash solutions, centrifuged solids, and total sample.

${ }^{4}$ Calculated assuming TOC is $100 \%$ oxalate in the centrifuged solids. 
The "Total Mass Calculated" in Table 7-2 and following tables represents the sum of the individual components in each fraction. The basic formula is:

$$
\text { Total Mass Calc }=\sum_{i} \mathrm{Mi}+3 \mathrm{MToc}+5 \mathrm{MTTC}+(59 / 27) \mathrm{M}_{\mathrm{Al}}
$$

where $M_{i}$ is the mass in grams of each metal ion ( $A 1$ through $U$ but not $P$ or $S$ ) and anion ( $\mathrm{F}^{-}$through $\mathrm{SO}_{4}{ }^{2-}$ plus $\mathrm{OH}^{-}$) and $\mathrm{H}_{2} \mathrm{O}$. Masses of TOC, TIC, and $\mathrm{Al}$ are adjusted to account for the weight of oxygen and other elements associated with the aluminum and carbon. The TOC multiplier is based on the molecular weight to carbon weight ratio of "typical" organic compounds likely to be present in the waste, such as acetate and chelating agents. The TIC multiplier converts carbon to carbonate. The Al multiplier converts $\mathrm{Al}$ to $\mathrm{AlO}_{2}^{-}$, the anhydrous form of the aluminate ion, $\mathrm{Al}(\mathrm{OH})_{4}^{-}$. The anhydrous form is used because the hydration water is included in the $\mathrm{H}_{2} \mathrm{O}$ mass as determined by TGA or oven-drying. No attempt was made to account for speciation of the more minor components.

In some cases, modifications to the basic formula are necessary. Where oxalate values are higher than the TOC would account for, the mass of oxalate is used instead of the adjusted mass of TOC. Where P values are higher than one-third of the corresponding phosphate values, the $\mathrm{P}$ mass is used and adjusted by the multiplier (95/31) to convert mass of $\mathrm{P}$ to mass of $\mathrm{PO}_{4}{ }^{3+}$.

The "Total Mass Measured" in Table 7-2 and following tables is based on actual weights of fractions (initial sample, wash solutions, and centrifuged solids) recorded during the centrifuge cone phase separations. These values are taken from Table 7-1.

The agreement between Total Mass Calculated and Total Mass Measured provides an estimate of the degree of reliability of the analytical results and the calculations performed. Agreement within $\pm 5 \%$ is considered good.

\subsubsection{Solid/Liquid Phase Distribution}

Table 7-3 (next page) is similar to Table 7-2. In Table 7-3, all results are "normalized" to 100 grams of composite sample. This table shows breakdown by solid/liquid phase distribution.

The first data column ("Total Sample") shows the amount of each analyte present in $100 \mathrm{~g}$ saltcake, regardless of whether it is present in the solid or liquid phase. The value is the same as that found in the "Wt\%" column in Table 7-2. The $\mathrm{H}_{2} \mathrm{O}$ entry in this column includes the $100 \mathrm{~g}$ dilution water added per $100 \mathrm{~g}$ saltcake.

The second data column ("Supernate") shows the weight of each component present in the supernatant liquid decanted from the sample at $100 \%$ by weight dilution. This is calculated by normalizing (dividing by total sample weight and multiplying by 100) the values from Table $7-2$ " 1 st Wash". Comparing this column with the previous one provides an estimate of how much of each component in the saltcake waste would be 
retrievable by pumping the liquid from the tank after a $1: 1$ dilution (by weight) with water at $25^{\circ} \mathrm{C}$.

Table 7-3. Tank BY-102 Solid/Liquid Phase Distribution at 100\% Dilution ${ }^{1}$ (all values in grams per 100 grams of saltcake sample)

\begin{tabular}{|c|c|c|c|c|c|c|}
\hline Analyte & $\begin{array}{c}\text { Total } \\
\text { Sample }\end{array}$ & Supernate & $\begin{array}{c}\text { Interstitial } \\
\text { Liquid }\end{array}$ & $\begin{array}{c}\text { Total } \\
\text { Liquid }\end{array}$ & $\begin{array}{c}\text { Undissolved } \\
\text { Solids }\end{array}$ & $\begin{array}{c}\% \% \\
\text { Undissolved }\end{array}$ \\
\hline $\mathrm{Al}$ & 1.65 & 0.90 & 0.28 & 1.18 & 0.46 & 28 \\
\hline $\mathrm{Ca}$ & 0.043 & & & & 0.043 & 100 \\
\hline $\mathrm{Cr}$ & 0.199 & 0.115 & 0.036 & 0.151 & 0.048 & 24 \\
\hline $\mathrm{Fe}$ & 0.053 & & & & 0.053 & 100 \\
\hline $\mathrm{K}$ & 0.136 & 0.136 & 0.043 & 0.179 & -0.043 & 0 \\
\hline $\mathrm{Mn}$ & 0.024 & & & & 0.024 & 100 \\
\hline $\mathrm{Na}$ & 27.75 & 16.12 & 5.09 & 21.21 & 6.54 & 24 \\
\hline $\mathrm{Ni}$ & 0.026 & & & & 0.026 & 100 \\
\hline $\mathrm{P}$ & 0.998 & 0.062 & 0.020 & 0.082 & 0.916 & 92 \\
\hline $\mathrm{S}$ & 2.03 & 0.37 & 0.12 & 0.49 & 1.55 & 76 \\
\hline $\mathrm{Si}$ & 0.072 & 0.027 & 0.008 & 0.035 & 0.037 & 51 \\
\hline $\mathrm{U}$ & 0.064 & & & & 0.064 & 100 \\
\hline $\mathrm{F}$ & 1.17 & 0.28 & 0.09 & 0.37 & 0.80 & 69 \\
\hline $\mathrm{Cl}^{-}$ & 0.11 & 0.11 & 0.035 & 0.14 & -0.035 & 0 \\
\hline $\mathrm{NO}_{2}{ }^{-}$ & 1.85 & 1.53 & 0.48 & 2.01 & -0.16 & 0 \\
\hline $\mathrm{NO}_{3}{ }^{-}$ & 10.92 & 9.54 & 3.01 & 12.55 & -1.63 & 0 \\
\hline $\mathrm{PO}_{4}^{3^{--}}$ & $(0.60)$ & 0.17 & 0.06 & 0.23 & $(0.37)$ & see $\mathrm{P}$ \\
\hline $\mathrm{SO}_{4}^{2-}$ & 5.12 & 1.08 & 0.34 & 1.43 & 3.69 & 72 \\
\hline $\mathrm{C}_{2} \mathrm{O}_{4}{ }^{2-}$ & 1.99 & 0.10 & 0.03 & 0.13 & 1.86 & 94 \\
\hline $\mathrm{TIC}$ & 4.13 & 3.38 & 1.07 & 4.45 & -0.32 & 0 \\
\hline TOC & 0.63 & 0.105 & 0.033 & 0.14 & 0.49 & 78 \\
\hline $\mathrm{OH}^{-}$ & 1.09 & 0.93 & 0.29 & 1.23 & -0.14 & 0 \\
\hline${ }^{137} \mathrm{Cs}(\mathrm{mCi})$ & 4.44 & 3.73 & 1.18 & 4.91 & -0.47 & 0 \\
\hline $\mathrm{H}_{2} \mathrm{O}$ & 126.51 & 91.30 & 35.21 & 126.51 & 0.00 & 0 (assumed) \\
\hline $\begin{array}{l}\text { Total Mass } \\
\text { Calculated }^{2}\end{array}$ & 204.3 & 140.5 & 50.8 & 190.1 & 18.9 & -- \\
\hline $\begin{array}{l}\text { Total Mass } \\
\text { Measured }^{3}\end{array}$ & 200.0 & 132.4 & 51.0 & 183.4 & 16.6 & -- \\
\hline
\end{tabular}

${ }^{1}$ Blank entries indicate analytical result was below detection limit.

${ }^{2}$ Sum of individual components (see text for details).

${ }^{3}$ Actual weights of wash solutions, centrifuged solids, and total sample.

${ }^{4}$ Calculated assuming TOC is $100 \%$ oxalate in the centrifuged solids. 
The next column shows how much of each component remains in the interstitial liquid of the centrifuged solids after the $I^{\text {st }}$ water wash. This is calculated by assuming that all of the water in the centrifuged solids is associated with interstitial liquid. This assumption is in error to the extent that some of the undissolved salts may contain waters of hydration. The values in this column represent a fraction of the corresponding Supernate column values, where the fraction is determined by the $\% \mathrm{H}_{2} \mathrm{O}$ analyses.

The "Total Liquid" column represents the sum of the two previous columns. "Undissolved Solids" is the Total Sample minus the Total Liquid. Negative numbers in this column point out obvious (but smail) errors in the data, which are probably related to the assumption used to assign the amount of interstitial liquid present.

The last column ("\%Undissolved) represents the fraction of each component that remains in the solid phase relative to the total amount of that component present in the sample.

\subsection{Tank BY-106}

Tables 7-4 through 7-6 have the same explanations as the corresponding tables for tank BY-102. Tank BY-106 saltcake contains a much higher percentage of $\mathrm{NaNO}_{3}$ than tank BY-102 saltcake, and is much more soluble at 100\% dilution. As shown in Tables $7-3$ and 7-6, the total amount of undissolved solids is approximately $3 \%$ of the initial sample weight for BY-106, compared to $17 \%$ for BY -102 .

Table 7-4. Summary of Measurements from BY-106 Sequential Dissolution Test (weights in grams, volumes in $\mathrm{mL}$ )

\begin{tabular}{|c|c|c|c|}
\hline Measurement & SEQA & SEQB & Total \\
\hline Sample Weight & 30.017 & 30.091 & 60.108 \\
\hline Wt $\mathrm{H}_{2} \mathrm{O}$ Added & 30.026 & 30.139 & 60.165 \\
\hline Wt $1^{\text {st }} \mathrm{H}_{2} \mathrm{O}$ Wash & 53.341 & 54.005 & 107.346 \\
\hline $\mathrm{Vol} \mathrm{1}{ }^{\mathrm{st}} \mathrm{H}_{2} \mathrm{O}$ Wash & 40.3 & 41.9 & 82.2 \\
\hline Density $1^{\mathrm{st}} \mathrm{H}_{2} \mathrm{O}$ Wash & & & 1.336 \\
\hline$\% \mathrm{H}_{2} \mathrm{O} 1^{\mathrm{st}} \mathrm{H}_{2} \mathrm{O}$ Wash & & & $58.2^{\mathrm{a}}$ \\
\hline Wt Centrifuged Solids & 6.702 & 6.225 & 12.927 \\
\hline Wt $\mathrm{H}_{2} \mathrm{O}$ Added & 29.440 & 31.020 & 60.460 \\
\hline $\mathrm{Wt} 2^{\text {nd }} \mathrm{H}_{2} \mathrm{O}$ Wash & 34.572 & 35.992 & 70.564 \\
\hline $\mathrm{Vol} 2^{\text {nd }} \mathrm{H}_{2} \mathrm{O}$ Wash & 31.0 & 32.5 & 63.5 \\
\hline Density $2^{\text {nd }} \mathrm{H}_{2} \mathrm{O}$ Wash & & & 1.087 \\
\hline$\% \mathrm{H}_{2} \mathrm{O} 2^{\text {nid }} \mathrm{H}_{2} \mathrm{O}$ Wash & & & 91.4 \\
\hline Final Wt Centrifuged Solids & 1.570 & 1.253 & 2.823 \\
\hline$\% \mathrm{H}_{2} \mathrm{O}$ Centrifuged Solids & & & 69.91 \\
\hline
\end{tabular}

a corresponds to $50 \% \mathrm{H}_{2} \mathrm{O}$ in centrifuged solids after $1^{\text {st }}$ wash (which wasn't measured) 
Table 7-5. Tank BY-106 Analysis by Fractions ${ }^{1}$ (all values in grams)

\begin{tabular}{|c|c|c|c|c|c|}
\hline Analyte & $1^{\text {st }}$ Wash & $2^{\text {nd }}$ Wash & CSol & Total & $\mathrm{Wt} \%$ \\
\hline $\mathrm{Al}$ & 0.85 & 0.07 & 0.04 & 0.95 & 1.59 \\
\hline $\mathrm{Ca}$ & & & 0.008 & 0.008 & 0.01 \\
\hline $\mathrm{Cr}$ & 0.020 & 0.002 & 0.046 & 0.068 & 0.11 \\
\hline $\mathrm{Fe}$ & & & 0.013 & 0.013 & 0.02 \\
\hline $\mathrm{K}$ & 0.136 & 0.010 & & 0.146 & 0.24 \\
\hline $\mathrm{Mn}$ & & & 0.001 & 0.001 & 0.00 \\
\hline $\mathrm{Na}$ & 12.90 & 1.87 & 0.24 & 15.01 & 25.0 \\
\hline $\mathrm{Ni}$ & & & 0.003 & 0.003 & 0.01 \\
\hline$P$ & 0.020 & 0.025 & 0.002 & 0.047 & 0.08 \\
\hline $\mathrm{S}$ & 0.077 & 0.185 & 0.007 & 0.268 & 0.45 \\
\hline $\mathrm{Si}$ & 0.007 & 0.002 & 0.001 & 0.010 & 0.02 \\
\hline $\mathrm{U}$ & 0.012 & & & 0.012 & 0.02 \\
\hline $\mathrm{F}$ & 0.17 & 0.20 & na & 0.37 & 0.62 \\
\hline $\mathrm{Cl}$ & 0.091 & 0.007 & na & 0.098 & 0.16 \\
\hline $\mathrm{NO}_{2}$ & 1.50 & 0.13 & na & 1.63 & 2.71 \\
\hline $\mathrm{NO}_{3}^{-}$ & 22.43 & 1.93 & na & 24.37 & 40.5 \\
\hline $\mathrm{PO}_{4}^{3-}$ & 0.12 & 0.08 & na & 0.20 & 0.34 \\
\hline $\mathrm{SO}_{4}{ }^{2-}$ & 0.14 & 0.56 & na & 0.70 & 1.16 \\
\hline $\mathrm{C}_{2} \mathrm{O}_{4}{ }^{2^{-}}$ & 0.04 & 0.47 & $0.25^{4}$ & 0.76 & 1.26 \\
\hline TIC & 0.79 & 0.07 & 0.004 & 0.86 & 1.43 \\
\hline TOC & 0.08 & 0.12 & 0.08 & 0.29 & 0.48 \\
\hline $\mathrm{OH}^{-}$ & 0.63 & 0.06 & na & 0.69 & 1.14 \\
\hline${ }^{737} \mathrm{Cs}(\mathrm{mCi})$ & 4.31 & 0.40 & na & 4.71 & $\cdots$ \\
\hline $\mathrm{H}_{2} \mathrm{O}$ & 62.48 & 64.50 & 1.97 & 8.84 & 14.7 \\
\hline $\begin{array}{l}\text { Total Mass } \\
\text { Calculated }^{2} \\
\end{array}$ & 106.7 & 70.3 & 3.0 & 59.6 & 99.1 \\
\hline $\begin{array}{l}\text { Total Mass } \\
\text { Measured }^{3}\end{array}$ & 107.3 & 70.6 & 2.8 & 60.1 & 100.0 \\
\hline
\end{tabular}

${ }^{1}$ Blank entries indicate analytical result was below detection limit.

${ }^{2}$ Sum of individual components (see text for details).

${ }^{3}$ Actual weights of wash solutions, centrifuged solids, and total sample.

${ }^{4}$ Calculated assuming TOC is $100 \%$ oxalate in the centrifuged solids. 
Table 7-6. Tank BY-106 Solid/Liquid Phase Distribution at $100 \%$ Dilution ${ }^{1}$ (all values in grams per 100 grams of saltcake sample)

\begin{tabular}{|c|c|c|c|c|c|c|}
\hline Analyte & $\begin{array}{c}\text { Total } \\
\text { Sample }\end{array}$ & Supernate & $\begin{array}{c}\text { Interstitial } \\
\text { Liquid } \\
\end{array}$ & $\begin{array}{l}\text { Total } \\
\text { Liquid }\end{array}$ & $\begin{array}{c}\text { Undissolved } \\
\text { Solids }\end{array}$ & $\begin{array}{c}\% \\
\text { Undissolved }\end{array}$ \\
\hline $\mathrm{Al}$ & 1.58 & 1.41 & 0.15 & 1.56 & 0.03 & 2 \\
\hline $\mathrm{Ca}$ & 0.013 & & & & 0.013 & 100 \\
\hline $\mathrm{Cr}$ & 0.113 & 0.034 & 0.003 & 0.037 & 0.076 & 67 \\
\hline $\mathrm{Fe}$ & 0.019 & & & & 0.019 & 100 \\
\hline $\mathrm{K}$ & 0.243 & 0.227 & 0.024 & 0.251 & -0.008 & 0 \\
\hline $\mathrm{Na}$ & 24.98 & 21.47 & 2.24 & 23.71 & 1.27 & 5 \\
\hline $\mathrm{Ni}$ & 0.006 & & & & 0.006 & 100 \\
\hline $\mathrm{P}$ & 0.078 & 0.033 & 0.003 & 0.036 & 0.042 & $5 \overline{4}$ \\
\hline $\mathrm{S}$ & 0.447 & 0.128 & 0.013 & 0.142 & 0.305 & 68 \\
\hline $\mathrm{Si}$ & 0.017 & 0.012 & 0.001 & 0.013 & 0.004 & 24 \\
\hline $\mathrm{U}$ & 0.019 & & & & 0.019 & 100 \\
\hline $\mathrm{F}$ & 0.62 & 0.28 & 0.03 & 0.32 & 0.30 & 49 \\
\hline $\mathrm{Cl}^{-}$ & 0.16 & 0.15 & 0.02 & 0.17 & -0.01 & 0 \\
\hline $\mathrm{NO}_{2}{ }^{-}$ & 2.71 & 2.49 & 0.26 & 2.75 & -0.04 & 0 \\
\hline $\mathrm{NO}_{3}$ & 40.54 & 37.32 & 3.89 & 41.22 & -0.68 & 0 \\
\hline $\mathrm{PO}_{4}{ }^{3^{-}}$ & 0.34 & 0.20 & 0.02 & 0.23 & 0.11 & 34 \\
\hline $\mathrm{SO}_{4}{ }^{2-}$ & 1.16 & 0.23 & 0.02 & 0.26 & 0.90 & 78 \\
\hline $\mathrm{C}_{2} \mathrm{O}_{4}{ }^{2^{-}}$ & 1.26 & 0.06 & 0.01 & 0.07 & 1.19 & 94 \\
\hline TIC & 1.43 & 1.31 & 0.14 & 1.45 & -0.02 & 0 \\
\hline TOC & 0.48 & 0.13 & 0.01 & 0.14 & 0.33 & 70 \\
\hline $\mathrm{OH}$ & 1.14 & 1.05 & 0.11 & 1.16 & -0.02 & 0 \\
\hline${ }^{137} \mathrm{Cs}(\mathrm{mCi})$ & 7.83 & 7.17 & 0.75 & 7.91 & -0.08 & 0 \\
\hline $\mathrm{H}_{2} \mathrm{O}$ & 114.70 & 103.95 & 10.75 & 114.70 & 0.00 & 0 (assumed) \\
\hline $\begin{array}{l}\text { Total Mass } \\
\text { Calculated }^{2}\end{array}$ & 199.2 & 177.6 & 18.4 & 196.1 & 4.0 & -- \\
\hline $\begin{array}{l}\text { Total Mass } \\
\text { Measured }^{3}\end{array}$ & 200.0 & 178.6 & 18.5 & 197.1 & 2.9 & -- \\
\hline
\end{tabular}

${ }^{1}$ Blank entries indicate analytical result was below detection limit.

${ }^{2}$ Sum of individual components (see text for details).

${ }^{3}$ Actual weights of wash solutions, centrifuged solids, and total sample.

${ }^{4}$ Calculated assuming TOC is $100 \%$ oxalate in the centrifuged solids. 


\subsection{Tank B-106}

Tables 7-7 through 7-9 have the same explanations as the corresponding tables for tank BY-102. Inhibited water $\left(0.01 \mathrm{M} \mathrm{NaOH}\right.$ and $\left.0.01 \mathrm{M} \mathrm{NaNO}_{2}\right)$ was used for the dilution rather than pure $\mathrm{H}_{2} \mathrm{O}$ because of the initial low $\mathrm{pH}(<12)$ of the composite sample. Tank B-106 waste has many of the properties of a sludge, as well as some properties of a saltcake. The initial high water content $\left(58 \% \mathrm{H}_{2} \mathrm{O}\right)$ and low pH are typical of a sludge. On the other hand, $\mathrm{NaNO}_{3}$ makes up a very high percentage of the non- $\mathrm{H}_{2} \mathrm{O}$ fraction of the waste, typical of a saltcake.

Table 7-7. Summary of Measurements from B-106 Sequential Dissolution Test with Inhibited Water Diluent

(weights in grams, volumes in $\mathrm{mL}$ )

\begin{tabular}{|c|c|c|c|}
\hline Measurement & SEQA & SEQB & Total \\
\hline Sample Weight & 25.712 & 25.999 & 51.711 \\
\hline Wt inh. $\mathrm{H}_{2} \mathrm{O}$ Added & 25.012 & 24.981 & 49.993 \\
\hline Wt $1^{\text {st }} \mathrm{H}_{2} \mathrm{O}$ Wash & 37.792 & 37.903 & 75.695 \\
\hline Vol $1^{\text {st }} \mathrm{H}_{2} \mathrm{O}$ Wash & 33.8 & 34.5 & 68.35 \\
\hline Density $1^{\text {st }} \mathrm{H}_{2} \mathrm{O}$ Wash & & & 1.107 \\
\hline$\% \mathrm{H}_{2} \mathrm{O}^{\text {st }} \mathrm{H}_{2} \mathrm{O}$ Wash & & & $84.1^{2}$ \\
\hline Wt Centrifuged Solids & 12.932 & 13.077 & 26.009 \\
\hline Wt inh. $\mathrm{H}_{2} \mathrm{O}$ Added & 24.974 & 25.061 & 50.035 \\
\hline Wt $2^{\text {nd }} \mathrm{H}_{2} \mathrm{O}$ Wash & 25.633 & 25.741 & 51.374 \\
\hline Vol $2^{\text {nd }} \mathrm{H}_{2} \mathrm{O}$ Wash & 25.1 & 25.0 & 50.1 \\
\hline Density $2^{\text {nat }} \mathrm{H}_{2} \mathrm{O}$ Wash & & & 1.023 \\
\hline$\% \mathrm{H}_{2} \mathrm{O} 2^{\text {nd }} \mathrm{H}_{2} \mathrm{O}$ Wash & & & 95.7 \\
\hline Final Wt Centrifuged Solids & 12.273 & 12.397 & 24.670 \\
\hline$\% \mathrm{H}_{2} \mathrm{O}$ Centrifuged Solids & & & 76.7 \\
\hline
\end{tabular}

a corresponds to $67 \% \mathrm{H}_{2} \mathrm{O}$ in centrifuged solids after $1^{\text {st }}$ wash (which wasn't measured)

Corresponding data for the dissolution in $3 \mathrm{M} \mathrm{NaOH}$ are not shown. Refer to Tables 3-14 and 3-15 for the analytical results. The $3 \mathrm{M} \mathrm{NaOH}$ enhanced the solubility of aluminum, which is a minor component, and depressed the solubility of phosphate, which is only sparingly soluble in either diluent. It had little or no effect on the major components, relative to inhibited water as the diluent. 
Table 7-8 Tank B-106 Analysis by Fractions, Inhibited Water Diluent ${ }^{1}$ (all values in grams)

\begin{tabular}{|c|c|c|c|c|c|}
\hline Analyte & $\mathrm{I}^{\text {st }}$ Wash & $2^{\text {nd }}$ Wash & CSol & Total & $\mathrm{Wt} \%$ \\
\hline $\mathrm{Al}$ & & & 0.12 & 0.12 & 0.23 \\
\hline $\mathrm{Bi}$ & & & 0.13 & 0.13 & 0.24 \\
\hline $\mathrm{Ca}$ & & & 0.12 & 0.12 & 0.23 \\
\hline $\mathrm{Cr}$ & 0.001 & & 0.009 & 0.010 & 0.02 \\
\hline $\mathrm{Fe}$ & & & 0.68 & 0.68 & 1.31 \\
\hline $\mathrm{K}$ & 0.007 & 0.001 & & 0.008 & 0.02 \\
\hline $\mathrm{Mg}$ & & & 0.029 & 0.029 & 0.06 \\
\hline $\mathrm{Na}$ & 3.17 & 0.69 & 1.05 & 4.91 & 9.49 \\
\hline $\mathrm{Ni}$ & & & 0.002 & 0.002 & 0.01 \\
\hline $\mathrm{P}$ & 0.23 & 0.06 & 0.69 & 0.98 & 1.90 \\
\hline $\mathrm{S}$ & 0.20 & 0.04 & 0.02 & 0.26 & 0.49 \\
\hline $\mathrm{Sr}$ & & & 0.018 & 0.018 & 0.04 \\
\hline $\mathrm{U}$ & & & 0.47 & 0.47 & 0.90 \\
\hline $\mathrm{F}^{-}$ & 0.036 & 0.027 & na & 0.063 & 0.12 \\
\hline $\mathrm{Cl}^{-}$ & 0.048 & 0.009 & na & 0.057 & 0.11 \\
\hline $\mathrm{NO}_{2}$ & 0.39 & 0.08 & na & 0.47 & 0.90 \\
\hline $\mathrm{NO}_{3}$ & 7.04 & 1.34 & na & 8.38 & 16.20 \\
\hline $\mathrm{PO}_{4}{ }^{3-}$ & 0.77 & 0.18 & na & 0.95 & 1.83 \\
\hline $\mathrm{SO}_{4}{ }^{2-}$ & 0.50 & 0.10 & na & 0.60 & 1.15 \\
\hline $\mathrm{C}_{2} \mathrm{O}_{4}{ }^{2-}$ & \multicolumn{5}{|c|}{ not detected in any fractions } \\
\hline $\mathrm{TIC}$ & 0.011 & 0.003 & 0.003 & 0.017 & 0.03 \\
\hline TOC & 0.014 & 0.003 & 0.009 & 0.026 & 0.05 \\
\hline $\mathrm{OH}^{-}$ & \multicolumn{5}{|c|}{ not detected in any fractions } \\
\hline${ }^{137} \mathrm{Cs}(\mathrm{mCi})$ & 0.15 & 0.025 & na & 0.175 & -- \\
\hline $\mathrm{H}_{2} \mathrm{O}$ & 63.66 & 49.16 & 18.92 & 29.99 & 58.00 \\
\hline $\begin{array}{l}\text { Total Mass } \\
\text { Calculated }^{2}\end{array}$ & 75.7 & 51.6 & 23.9 & 49.4 & 97.1 \\
\hline $\begin{array}{l}\text { Total Mass } \\
\text { Measured }^{3}\end{array}$ & 75.7 & 51.4 & 24.7 & 51.7 & 100.0 \\
\hline
\end{tabular}

${ }^{1}$ Blank entries indicate analytical result was below detection limit.

${ }^{2}$ Sum of individual components (see text for details).

${ }^{3}$ Actual weights of wash solutions, centrifuged solids, and total sample. 
Table 7-9. Tank B-106 Solid/Liquid Phase Distribution at 100\% Dilution ${ }^{1}$ with Inhibited Water Diluent

(all values in grams per 100 grams of saltcake sample)

\begin{tabular}{|c|c|c|c|c|c|c|}
\hline Analyte & $\begin{array}{c}\text { Total } \\
\text { Sample }\end{array}$ & Supernate & $\begin{array}{l}\text { Interstitial } \\
\text { Liquid }\end{array}$ & $\begin{array}{c}\text { Total } \\
\text { Liquid }\end{array}$ & $\begin{array}{l}\text { Undissolved } \\
\text { Solids }\end{array}$ & $\begin{array}{c}\% \\
\text { Undissolved }\end{array}$ \\
\hline $\mathrm{Al}$ & 0.23 & & & & 0.23 & 100 \\
\hline $\mathrm{Bi}$ & 0.25 & & & & 0.25 & 100 \\
\hline $\mathrm{Ca}$ & 0.24 & & & & 0.24 & 100 \\
\hline $\mathrm{Cr}$ & 0.020 & 0.002 & 0.001 & 0.003 & 0.018 & 87 \\
\hline $\mathrm{Fe}$ & 1.31 & & & & 1.31 & 100 \\
\hline $\mathrm{K}$ & 0.016 & 0.014 & 0.004 & 0.017 & -0.001 & 0 \\
\hline $\mathrm{Mg}$ & 0.057 & & & & 0.057 & 100 \\
\hline $\mathrm{Na}$ & 9.49 & 6.13 & 1.64 & 7.78 & 1.71 & 18 \\
\hline $\mathrm{Ni}$ & 0.004 & & & & 0.004 & 100 \\
\hline $\mathrm{P}$ & 1.90 & 0.45 & 0.12 & 0.57 & 1.33 & 70 \\
\hline$S$ & 0.49 & 0.38 & 0.10 & 0.48 & 0.01 & 2 \\
\hline $\mathrm{Si}$ & 0.018 & $0.00 \mathrm{~L}$ & & 0.001 & 0.017 & 94 \\
\hline $\mathrm{Sr}$ & 0.034 & & & & 0.034 & 100 \\
\hline U & 0.91 & & & & 0.91 & 100 \\
\hline $\mathrm{F}^{-}$ & 0.12 & 0.07 & 0.02 & 0.09 & 0.03 & 27 \\
\hline $\mathrm{Cl}^{-}$ & 0.11 & 0.09 & 0.02 & 0.12 & -0.01 & 0 \\
\hline $\mathrm{NO}_{2}{ }^{-}$ & 0.91 & 0.76 & 0.20 & 0.96 & -0.05 & 0 \\
\hline $\mathrm{NO}_{3}{ }^{-}$ & 16.20 & 13.62 & 3.65 & 17.27 & -1.06 & 0 \\
\hline $\mathrm{PO}_{4}{ }^{3-}$ & $(1.83)$ & 1.49 & 0.40 & 1.90 & \multicolumn{2}{|c|}{ see $\mathrm{P}$} \\
\hline $\mathrm{SO}_{4}{ }^{2-}$ & 1.15 & 0.96 & 0.26 & 1.21 & -0.06 & 0 \\
\hline TIC & 0.033 & 0.021 & 0.006 & 0.027 & 0.006 & 18 \\
\hline TOC & 0.051 & 0.027 & 0.007 & 0.034 & 0.017 & 33 \\
\hline${ }^{137} \mathrm{Cs}(\mathrm{mCi})$ & 0.34 & 0.29 & 0.08 & 0.37 & -0.03 & 0 \\
\hline $\mathrm{H}_{2} \mathrm{O}$ & 158.01 & 123.12 & 34.88 & 158.01 & 0.00 & 0 (assumed) \\
\hline $\begin{array}{l}\text { Total Mass } \\
\text { Calculated }^{2}\end{array}$ & 195.8 & 146.5 & 41.1 & 187.6 & 10.2 & -- \\
\hline $\begin{array}{l}\text { Total Mass } \\
\text { Measured }^{3}\end{array}$ & 200.0 & 146.4 & 41.5 & 187.9 & 12.1 & -- \\
\hline
\end{tabular}

${ }^{1}$ Blank entries indicate analytical result was below detection limit.

${ }^{2}$ Sum of individual components (see text for details).

${ }^{3}$ Actual weights of wash solutions, centrifuged solids, and total sample.

${ }^{4}$ Calculated assuming TOC is $100 \%$ oxalate in the centrifuged solids. 


\subsection{COMPARISONS}

This section is devoted to comparisons of the current data with computer modeling predictions, with Tank Characterization Report (TCR) characterization data, and with prior sludge washing studies.

\subsection{COMPUTER MODELING}

Computer simulations of the saltcake series dissolution tests for tanks BY-102 and BY-106 were conducted by investigators at Mississippi State University using the Environmental Simulation Program (ESP). The full results of the modeling will be published by them in the near future. A brief summary is provided here.

Analytical results from the sequential dissolution experiments were used as input to the ESP program. All anions from the analysis were included, and all cations present at concentrations $>150 \mathrm{ppm}$ were included in the simulation for each tank waste studied. Limits imposed on the number of interphase and intraphase equilibrium relationships which can be considered by ESP necessitated the exclusion of cations present at low concentrations. The input data were reconciled for electroneutrality and for $\mathrm{pH}$. The result of these reconciliation processes was a stream with the molecular composition which reproduced the measured analytical data.

Dilution ratios of $0 \%$ to $500 \%$ by weight $(100 \%$ dilution $=1 \mathrm{~g}$ saltcake $/ \mathrm{g}$ diluent) were considered. For tanks BY-102 and BY-106, dilution with water at $25^{\circ} \mathrm{C}$ and $50^{\circ} \mathrm{C}$ were simulated. For both tanks, major trends in the experimental data were seen in the simulation results. At dilution ratios of $100 \%$ and higher, quantitative prediction was obtained for all major anions $\left(\mathrm{Cl}^{-}, \mathrm{NO}_{2}^{-}, \mathrm{NO}_{3}{ }^{-}, \mathrm{C}_{2} \mathrm{O}_{4}{ }^{2-}, \mathrm{F}^{-}, \mathrm{PO}_{4}{ }^{3-}, \mathrm{SO}_{4}{ }^{2-}\right.$, TIC). At dilution ratios of less than $100 \%$, quantitative prediction was obtained only for select anions $\left(\mathrm{Cl}^{-}, \mathrm{NO}_{2}^{-}, \mathrm{C}_{2} \mathrm{O}_{4}^{2-}, \mathrm{TIC}\right)$.

The major salt predicted in the solid phase at $0 \%$ dilution is sodium nitrate. A maximum in the nitrate concentration is predicted at ca. $40 \%$ dilution by simulation. The nitrate concentration then exhibits classic exponential decay at higher dilution ratios. Other species predicted to be present in the solid phase at $0 \%$ dilution include $\mathrm{Na}_{7} \mathrm{~F}\left(\mathrm{PO}_{4}\right)_{2}{ }_{1} 19 \mathrm{H}_{2} \mathrm{O}$ and $\mathrm{Na}_{3} \mathrm{FSO}_{4}$. The anions associate with these species showed marked discrepancies between the concentrations predicted by ESP and those measured experimentally at dilution ratios $<100 \%$. This indicates a need to further examine the solid-liquid equilibrium behavior for these double salt systems through well-defined laboratory experiments. Also beneficial in this effort would be speciation data on the solid phase, verifying that these double salts are indeed present in the undissolved saltcake. 


\subsection{COMPARISONS WITH TCR DATA AND SLUDGE WASH DATA}

Tank Characterization Reports (TCRs) have been published for all three tanks. The following tables provide comparisons of the current data with the core sample characterization data from the TCRs. For tank B-106, comparisons are also made with data from prior sludge washing tests.

In general, the agreement between the current data and TCR data is very good, but there are some differences. There are two reasons for these discrepancies - analytical errors and genuine sample differences.

The TCR data are typically averages of a large number of analytical determinations. Current data are single determinations or, at best, an average of two determinations. As a rule of thumb, analytical values are generally considered accurate to approximately $\pm 10 \%$. Larger differences between current data and TCR data are likely due to genuine differences in sample composition. These differences may indicate the variations in composition likely to be encountered within the tank waste during retrieval operations.

Composite samples for the current study were derived from a limited number of archived core segment samples. There is no guarantee that these composite samples accurately represent the overall tank waste composition. However, the solubility studies are at least internally consistent, because all of the tests for each tank were done on a single homogenized composite sample.

\subsubsection{Tank BY-102}

Table 8-1 shows the comparison between the TCR data (Sasaki 1997) and the current data (Total Sample column in Table 7-3) derived from the sequential dissolution tests. The TCR data are taken from Table B3-4 of the TCR, and represent the mean concentrations for solid segment sample data from the 1996 core samples.

The TCR data for metal ions are derived from fusion digest sample preparations, while the current data are derived from acid digest preparations. That might explain why certain metals are significantly higher in the TCR data (Al, Si). In general, the agreement between the two sets of data is very good, especially for the major components. 
Table 8-1. Tank BY-102 Comparison with TCR Data

(all values in grams per 100 grams of saltcake sample, i.e., weight percent)

\begin{tabular}{|c|c|c|}
\hline Analyte & Current Study & TCR \\
\hline $\mathrm{Al}$ & 1.65 & 4.16 \\
\hline $\mathrm{Ca}$ & 0.043 & $<0.21$ \\
\hline $\mathrm{Cr}$ & 0.199 & 0.187 \\
\hline $\mathrm{Fe}$ & 0.053 & $<0.19$ \\
\hline $\mathrm{Mn}$ & 0.024 & $<0.037$ \\
\hline $\mathrm{Na}$ & 27.75 & 26.7 \\
\hline $\mathrm{Ni}$ & 0.026 & $0.48^{1}$ \\
\hline $\mathrm{P}$ & 0.998 & $<0.95$ \\
\hline $\mathrm{S}$ & 2.03 & 1.73 \\
\hline $\mathrm{Si}$ & 0.072 & 0.435 \\
\hline $\mathrm{U}$ & 0.064 & $<1.01$ \\
\hline $\mathrm{F}$ & 1.17 & 1.80 \\
\hline $\mathrm{Cl}^{-}$ & 0.11 & 0.12 \\
\hline $\mathrm{NO}_{2}{ }^{-}$ & 1.85 & 1.39 \\
\hline $\mathrm{NO}_{3}^{-}$ & 10.92 & 9.50 \\
\hline $\mathrm{PO}_{4}{ }^{3-}$ & $(0.60)$ & 2.70 \\
\hline $\mathrm{SO}_{4}{ }^{2+}$ & 5.12 & 5.77 \\
\hline $\mathrm{C}_{2} \mathrm{O}_{4}{ }^{{ }^{-2}}$ & 1.99 & 1.93 \\
\hline TIC & 4.13 & 2.78 \\
\hline TOC & 0.63 & 0.44 \\
\hline $\mathrm{H}_{2} \mathrm{O}$ & 26.51 & 26.5 \\
\hline
\end{tabular}

${ }^{1}$ fusion in nickel crucible?

\subsubsection{Tank BY-106}

Table 8-2 shows the comparison between the TCR data (Bell 1996) and the current data (Total Sample column in Table 7-6) derived from the sequential dissolution tests. The TCR data are taken from Table 4-2 of the TCR, and represent the mean concentrations for data from the 1994-6 core samples 64, 65, and 121. Core 121 provided all of the segment samples used to make the composite sample for the current tests (see Table 2-1).

The current data tend to be higher than the corresponding TCR data, with some exceptions. The $\% \mathrm{H}_{2} \mathrm{O}$ is significantly lower for the current study, which indicates that the segment samples may have dried out somewhat during storage in the hotcells. Such drying would tend to make the other analytes appear relatively high in concentration. 
Table 8-2. Tank BY-106 Comparison with TCR Data (all values in grams per 100 grams of saltcake sample, i.e., weight percent)

\begin{tabular}{|c|c|c|}
\hline Analyte & Current Study & TCR \\
\hline $\mathrm{Al}$ & 1.58 & $2.04(2.87)^{1}$ \\
\hline $\mathrm{Ca}$ & 0.013 & 0.031 \\
\hline $\mathrm{Cr}$ & 0.113 & $0.086(0.13)$ \\
\hline $\mathrm{Fe}$ & 0.019 & 0.022 \\
\hline $\mathrm{K}$ & 0.243 & 0.247 \\
\hline $\mathrm{Na}$ & 24.98 & $20.3(21.1)$ \\
\hline $\mathrm{Ni}$ & 0.006 & 0.005 \\
\hline $\mathrm{P}$ & 0.078 & 0.103 \\
\hline $\mathrm{S}$ & 0.447 & 0.328 \\
\hline $\mathrm{Si}$ & 0.017 & 0.018 \\
\hline $\mathrm{U}$ & 0.019 & 0.016 \\
\hline $\mathrm{F}$ & 0.62 & 0.51 \\
\hline $\mathrm{Cl}^{-}$ & 0.16 & 0.21 \\
\hline $\mathrm{NO}_{2}{ }^{-}$ & 2.71 & 3.21 \\
\hline $\mathrm{NO}_{3}{ }^{-}$ & 40.54 & 32.9 \\
\hline $\mathrm{PO}_{4}{ }^{-2}$ & 0.34 & 0.53 \\
\hline $\mathrm{SO}_{4}{ }^{2-}$ & 1.16 & 1.13 \\
\hline $\mathrm{C}_{2} \mathrm{O}_{4}{ }^{2-}$ & 1.26 & 0.90 \\
\hline $\mathrm{TIC}^{\left.\mathrm{TOC}^{137} \mathrm{Cs}^{2} \mathrm{mCi}\right)}$ & 1.43 & 0.74 \\
\hline $\mathrm{H}_{2} \mathrm{O}$ & 0.48 & 0.25 \\
\hline & 7.83 & 10.6 \\
\hline & 14.70 & 25.5 \\
\hline
\end{tabular}

${ }^{1}$ fusion results in parentheses; others are from acid digest 


\subsubsection{Tank B-106}

Table 8-3 shows the comparison between the current data (Total Sample column in Table 7-9) derived from the sequential dissolution tests in inhibited water, TCR data (McCain 1996), and the data from sludge washing tests done at Los Alamos National Laboratory (Temer 1997). The TCR data are taken from Table ES-2 of the TCR, and represent the mean concentrations for segment sample data from the 1995 core samples 93 and 94 . The Temer data are taken from Table 4, page 74, in that report. The Temer data are reported as "concentration in initial solids", implying that the values might be adjusted to account for moisture content.

The waste contained two obvious layers that differed in physical appearance and in chemical composition. Each core sample contained two segments. The top segment (1) from each core and the top half of the bottom segment (2) were assigned to the "top layer", and the bottom half of the bottom segment was assigned to the "bottom layer". The composite sample for the current study contained portions of segment 1 upper and lower halves, and segment 2 upper half only. Therefore, the composite sample from the current study should represent only the "top layer" of waste in the tank. The data comparison indicates, however, that the composite sample may contain some of the bottom layer waste. The composite sample for the Temer study included samples from both waste layers. 
Table 8-3. Tank B-106 Comparison with TCR Data

(all values in grams per 100 grams of saltcake sample, i.e., weight percent)

\begin{tabular}{|c|c|c|c|c|}
\hline \multirow{2}{*}{ Analyte } & \multirow{2}{*}{ Current Study } & \multicolumn{2}{|c|}{ TCR } & \multirow{2}{*}{$\begin{array}{c}\text { Temer } \\
(1997)^{2}\end{array}$} \\
\hline & & Top Layer & Bottom Layer & \\
\hline $\mathrm{Al}$ & 0.23 & 0.15 & 1.37 & 0.73 \\
\hline $\mathrm{Bi}$ & 0.25 & 0.08 & 1.94 & 0.55 \\
\hline $\mathrm{Ca}$ & 0.24 & 0.24 & 0.03 & 0.53 \\
\hline $\mathrm{Cr}$ & 0.020 & 0.022 & 0.073 & 0.070 \\
\hline $\mathrm{Fe}$ & 1.31 & 1.84 & 8.22 & 3.42 \\
\hline $\mathrm{Na}$ & 9.49 & 10.7 & 12.1 & 19.7 \\
\hline $\mathrm{Ni}$ & 0.004 & 0.007 & 0.002 & 0.013 \\
\hline $\mathrm{Si}$ & 0.018 & 0.15 & 0.44 & 0.30 \\
\hline $\mathrm{Sr}$ & 0.034 & 0.051 & 0.015 & 0.097 \\
\hline $\mathrm{U}$ & 0.91 & 1.64 & 0.33 & 3.06 \\
\hline $\mathrm{F}^{-}$ & 0.12 & 0.15 & 1.01 & na \\
\hline $\mathrm{Cl}^{-}$ & 0.11 & 0.15 & 0.15 & na \\
\hline $\mathrm{NO}_{2}$ & 0.91 & 0.78 & 0.66 & na \\
\hline $\mathrm{NO}_{3}{ }^{-}$ & 16.20 & 19.4 & 17.0 & na \\
\hline $\mathrm{PO}_{4}{ }^{32}$ & $5.82^{1}$ & 7.56 & 6.71 & $\mathrm{na}$ \\
\hline $\mathrm{SO}_{4}{ }^{2-}$ & 1.15 & 1.48 & 1.40 & na \\
\hline TOC & 0.051 & 0.24 & 0.021 & na \\
\hline${ }^{137} \mathrm{Cs}(\mathrm{mCi})$ & 0.34 & 0.22 & 0.12 & na \\
\hline $\mathrm{H}_{2} \mathrm{O}$ & 58.01 & 62.4 & 56.8 & na \\
\hline
\end{tabular}

${ }^{1}$ calculated from $P$ result in Table 7-9.

${ }^{2}$ reported as "concentration in initial solids", rather than initial sludge.

The current data for the percentage of each analyte undissolved in the inhibitedwater wash (Table 7-9, last column) can be compared with similar data from Temer. The first step in the sludge wash experiments was an inhibited-water wash at $200 \%$ dilution ( $2 \mathrm{~g} \mathrm{H}_{2} \mathrm{O} / \mathrm{g}$ sludge) at $100{ }^{\circ} \mathrm{C}$ for 1 hour. The current study used $100 \%$ dilution at ambient temperature for 24 hours. The percentage of each analyte remaining in the solid phase under these conditions is shown in Table 8-4. 
Table 8-4. Percentage of B-106 Analyte Undissolved in Inhibited Water.

\begin{tabular}{|c|c|c|}
\hline Analyte & Current Study $^{1}$ & Temer $^{(1997)^{2}}$ \\
\hline $\mathrm{Al}$ & 100 & 72 \\
\hline $\mathrm{Bi}$ & 100 & 100 \\
\hline $\mathrm{Ca}$ & 100 & 100 \\
\hline $\mathrm{Cr}$ & 87 & 88 \\
\hline $\mathrm{Fe}$ & 100 & 100 \\
\hline $\mathrm{K}$ & 0 & 32 \\
\hline $\mathrm{Mg}$ & 100 & 100 \\
\hline $\mathrm{Na}$ & 18 & 5 \\
\hline $\mathrm{Ni}$ & 100 & 99 \\
\hline $\mathrm{P}$ & 70 & 40 \\
\hline $\mathrm{Si}$ & 94 & 95 \\
\hline $\mathrm{Sr}$ & 100 & 100 \\
\hline $\mathrm{U}$ & 100 & 99 \\
\hline
\end{tabular}

${ }^{1} 100 \%$ by weight dilution, ambient temperature, 24 hour mix time. $2200 \%$ by weight dilution, $100{ }^{\circ} \mathrm{C}, \mathrm{I}$ hour mix time. 


\subsection{References}

Agnew, S. F. (1997), J. Boyer, R. A. Corbin, R. B. Duran, J. R. FitzPatrick, K. A. Jurgensen, T. P. Ortiz, and B. L. Young, Hanford Tank Chemical and Radionuclide Inventories: HDW Model Rev. 4, LA-UR-96-3860, Los Alamos National Laboratory, Los Alamos, New Mexico.

Barney, G. S. (1997), The Solubilities of Significant Organic Compounds in HLW Tank Supernate Solutions - FY 1997 Progress Report, HNF-EP-0899-2, B\&W Hanford Company, Richland, Washington.

Beahm, E. C. (1998), C. F. Weber, D. D. Lee, T. A. Dillow, R. D. Hunt, C. M. Keswa, K. Osseo-Asare, and K. E. Spear, Status Report on Solid Control in Leachates, ORNL/TM-13660, Oak Ridge National Laboratory, Oak Ridge, Tennessee.

Bell, K. E. (1996), J. Franklin, J. Stroup and J. L. Huckaby, Tank Characterization Report for Single-Shell Tank 24I-BY-106, WHC-SD-WM-ER-616, Rev. 0, Westinghouse Hanford Company, Richland, Washington.

Herting, D. L. (1992), Identification of Crystals in Hanford Nuclear Waste Using Polarized Light Microscopy, WHC-MR-0375, Westinghouse Hanford Company, Richland, Washington.

Herting, D. L. (1996), Clean Salt Process Final Report, WHC-EP-0915, Westinghouse Hanford Company, Richland, Washington.

Herting, D. L. (1997), Results of Dihtion Studies with Waste from Tank 241-AN-105, HNF-SD-WM-DTR-046, Rev. 0, Numatec Hanford Corporation, Richland, Washington.

Herting, D. L. (1998), Results of Dihution Studies with Waste from Tank 241-AN-104, HNF-3352, Rev. 0, Numatec Hanford Corporation, Richland, Washington.

McCain, D. J. (1996), B. C. Simpson and L. C. Amato, Tank Characterization Report for Single-Shell Tank 241-B-106, WHC-SD-WM-ER-601, Rev. 0, Westinghouse Hanford Company, Richland, Washington.

Person, J. C. (1997), Effects of NaOH Dilution on Solution Concentrations in Tank 241-SY-101 Waste, WHC-SD-WM-DTR-045, Rev. 0, Numatec Hanford Corporation, Richland, Washington.

Sasaki, L. M. (1997), M. J. Kupfer, L. C. Amato, B. J. Morris, J. L. Stroup, R. D. Cromar and R. T. Winward, Tank Characterization Report for Single-Shell Tank 241-BY-102, HNF-SD-WM-ER-630, Rev. 0, Lockheed Martin Hanford Corporation, Richland, Washington. 
DISTRIBUTION SHEET

\begin{tabular}{|c|c|c|c|c|c|}
\hline \multirow{2}{*}{$\begin{array}{l}\text { To } \\
\text { Distribution }\end{array}$} & \multirow{2}{*}{\multicolumn{3}{|c|}{$\begin{array}{l}\text { From } \\
\text { NHC Process Chemistry }\end{array}$}} & \multicolumn{2}{|l|}{ Page 1 of 2} \\
\hline & & & & \multicolumn{2}{|c|}{ Date $09 / 30 / 98$} \\
\hline \multirow{2}{*}{\multicolumn{4}{|c|}{$\begin{array}{l}\text { Project Title/Work Order } \\
\text { Saltcake Dissolution FY } 1998 \text { Status Report }\end{array}$}} & \multicolumn{2}{|c|}{ EDT No. 625405} \\
\hline & & & & \multicolumn{2}{|l|}{ ECN No. NA } \\
\hline Name & MSIN & $\begin{array}{l}\text { Text } \\
\text { With All } \\
\text { Attach. }\end{array}$ & Text Only & $\begin{array}{l}\text { Attach./ } \\
\text { Appendix } \\
\text { Only }\end{array}$ & $\begin{array}{l}\text { EDT/ECN } \\
\text { Only }\end{array}$ \\
\hline
\end{tabular}

Numatec Hanford Corporation

Ba]dwin, J.H.

Bourges, J.

Certa, P.J.

Choho, A.F.

Flament, T.A

Gibbons, P.W.

Herting, D.L. (10)

Jewett, J.R.

Kirkbride, R.A.

Orme, R.M.

$\begin{array}{ll}\text { R3 } 3-73 & X \\ \text { T6-07 } & X \\ \text { R3-73 } & X \\ \text { R3-47 } & X \\ \text { H7 }-20 & X \\ \text { R3-74 } & X \\ \text { T6-07 } & X \\ \text { T6-07 } & X \\ \text { R3 }-73 & X \\ \text { R3-73 } & X \\ \text { T6-07 } & X\end{array}$

Fluor Daniel Hanford

Foreman, S.K. (5)

K9-46 $\quad X$

Lockheed Mart in Hanford Company

Crawford, T.W.

Jensen, R.D.

Kupfer, M.J.

Reynolds, D.A.

R3-73

R3 -75

R3-75

R2-11

$x$

$x$

$x$

$x$

$x$

$x$

$x$

$x$

$x$

$\hat{x}$

SESC

MacLean, G.T.

Place, D.E.

R3-73

R3-73

$x$

PNNL

Brooks, K.P.

Brouns, T.M.

$K 6-24 \quad X$

$K 9-91 \quad x$

$\mathrm{K} 8-93 \quad \mathrm{X}$

P7-25 $X$

$\mathrm{K} 8-96 \quad \mathrm{X}$

P7-25 $X$

K9-69 X

Notch, G.C. (5)

SAIC

Swanberg, D.J.

H0-50 X

Other

Kovach, J.L.

K6-51

$x$

Offsite

E.C. Beahm, Oak Ridge National Laboratory

P.0. Box 2008, Oak Ridge, TN 37831-6223 
Jimmy Be11, 137 Bowsprit Lane, Kingston, TN $37763 \times$

R.D. Hunt, Oak Ridge National Laboratory $X$

P.0. Box 2008, 0ak Ridge, TN 37831-6223

Jeff Lindner, Mississippi State University $X$

Campus Mailstop 9550, Etheredge Engineering B1dg,

Room 320, Box MM, Mississippi State, MS 39762

C.P. McGinnis, Oak Ridge National Laboratory

P.0. Box 2008, Oak Ridge, TN 37831-6223

Wally Schulz, W2S Company, Inc.

5314 Arbustos Court, NE, A1buquerque, NM 87111

John Swanson, 1318 Cottonwood Drive,

$X$

Richland, WA 99352

Don Temer, Los Alamos National Laboratory

$x$

NMT-1 CMR, MS G740, Los Alamos, NM 87545

Major Thompson, Westinghouse Savannah River

$X$

Company, Building C-140, Room 773-A, Savannah

$X$

$x$

$X$

River Technology Center, Aiken, SC 29808

Rebecca Toghiani, Mississippi State University $x$ Campus Mailstop 9595, Etheredge Engineering Building, Room 129, Box MM, Mississippi State, MS 39762

George Vandegrift, Argonne National Laboratory, $X$ 9700 South Cass Ave, B7dg 205, Argonne, IL 60439

J.S. Watson, Oak Ridge Natjonal Laboratory

$X$

P.0. Box 2008, 0ak Ridge, TN 37831-6223

T.D. Welch, Oak Ridge National Laboratory

P.0. Box 2008, Oak Ridge, TN 37831-6223 